\title{
CONFIRMATION OF THE \\ NON-EXISTENCE OF A PROJECTIVE PLANE OF ORDER 10
}

\author{
Dominique J. Roy \\ B. Math (Honours), Carleton University, 2002 \\ B. Sc., Carleton University, 2000 \\ A Thesis submitted to the \\ Faculty of Graduate Studies and Research \\ Carleton University \\ in partial fulfillment of the requirements for the \\ Master's degree in the \\ Ottawa-Carleton Institute for Graduate Studies and Research in \\ Mathematics and Statistics
}

C2010 Dominique J. Roy 


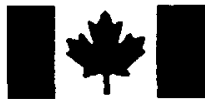

Library and Archives

Canada

Published Heritage Branch

395 Wellington Street

Ottawa ON K1A ON4

Canada
Bibliotheque et

Archives Canada

Direction du

Patrimoine de l'édition

395 , rue Wellington

Ottawa ON K1A ON4

Canada
Your file Votre référence
ISBN: 978-0-494-79585-9
Our file Notre référence
ISBN: $978-0-494-79585-9$

\section{NOTICE:}

The author has granted a nonexclusive license allowing Library and Archives Canada to reproduce, publish, archive, preserve, conserve, communicate to the public by telecommunication or on the Internet, loan, distribute and sell theses worldwide, for commercial or noncommercial purposes, in microform, paper, electronic and/or any other formats.

The author retains copyright ownership and moral rights in this thesis. Neither the thesis nor substantial extracts from it may be printed or otherwise reproduced without the author's permission.
AVIS:

L'auteur a accordé une licence non exclusive permettant à la Bibliothèque et Archives Canada de reproduire, publier, archiver, sauvegarder, conserver, transmettre au public par télécommunication ou par l'Internet, prêter, distribuer et vendre des thèses partout dans le monde, à des fins commerciales ou autres, sur support microforme, papier, électronique et/ou autres formats.

L'auteur conserve la propriété du droit d'auteur et des droits moraux qui protège cette thèse. Ni la thèse ni des extraits substantiels de celle-ci ne doivent être imprimés ou autrement reproduits sans son autorisation.
In compliance with the Canadian Privacy Act some supporting forms may have been removed from this thesis.

While these forms may be included in the document page count, their removal does not represent any loss of content from the thesis.
Conformément à la loi canadienne sur la protection de la vie privée, quelques formulaires secondaires ont été enlevés de cette thèse.

Bien que ces formulaires aient inclus dans la pagination, il n'y aura aucun contenu manquant.

\section{Canadä}




\section{Abstract}

Mathematicians have always been fascinated with existence problems. Fermat's Last Theorem ${ }^{\Psi}$ for example, was posed approximately 300 years before it was finally solved by Andrew Wiles just a few years short of the $21^{\text {st }}$ century, but not before dozens of talented mathematicians (and thousands of not so talented ones) had failed in their attempts to solve it. Another long-standing existence problem, solved just a few years before Fermat's, is the main subject of this thesis: does there exist a projective plane of order 10 ?

We begin laying the necessary groundwork by introducing projective planes and error-correcting codes. Next, a relationship between projective planes and codes is presented as the basis of a method to settle the existence of a projective plane of order 10 (unfortunately in the negative). We also give a historical account of the application of the solution. Finally, a confirmation of the non-existence is implemented and presented.

\footnotetext{
${ }^{\Psi} ? \exists n>2, x^{n}+y^{n}=z^{n}, x, y, z, n \in \mathbb{Z}$
} 


\section{Acknowledgements}

First off I would like to thank my supervisor, Brett Stevens, for his guidance, his patience in allowing me to pursue an extremely flexible course of part-time studies, and a (currently impossible) question ${ }^{\Psi}$ he posed in my first graduate course which led to my interest (okay, obsession) with finite projective planes.

I would also like to thank Cyril Garner for first sparking my interest in geometry: without his excellent lectures and ability to effectively impart his vast knowledge of, and passion for, the quirky world of non-Euclidean geometries, nor his encouragement to pursue a Master's degree, I may have never embarked on this long but satisfying journey.

A special thanks goes to Clement Lam for encouraging me to pursue this specific thesis and for giving me key advice along the way, and to David Erbach for pointing me in the right direction on solving the weight enumerator.

All professors encountered during my post-secondary studies have contributed to this thesis in some way. In particular, I would like to thank (in chronological order) Ann Hamilton, Greg Brown, F. Fiala, Mark Lanthier, Evangelos Kranakis, Maurice

\footnotetext{
${ }^{\Psi}$ Find a projective plane of order 12
} 
Chacron, Ken Williams, C.K. Fong, Robert Stanton, Luis Ribes, John Poland, John Dixon, Saban Alaca, Lucia Moura, Franz Oppacher and Daniel Panario.

I would like to thank my employer, Canada Revenue Agency, and the following supervisors for allowing me the flexibility and support to pursue university studies part-time: Gord McKenny, Gregg Stepto, Duane Locke, Margaret Cody, Sarah Wilson, William Prickett, Martin Lebeau, Mike Wolff and Brenda Goudie.

Finally, I would like to thank my parents for having instilled in me, through nature and/or nurture, a fundamental curiosity about all things in the world.

D.J. Roy 


\section{Contents}

Abstract ii

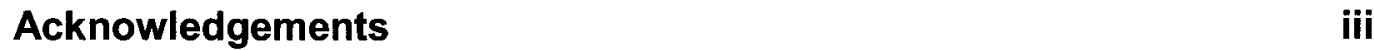

1 Background 1

1.1 Projective Planes........................................... 1

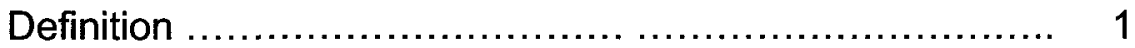

Balanced Incomplete Block Design ...................... 3

Latin Square............................................. 5

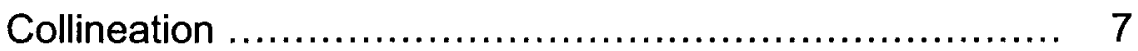

Incidence Matrix ...................................... 9

Search Complexity ................................... 9

1.2 Error Correcting Codes ..................................... 21

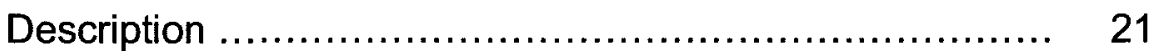

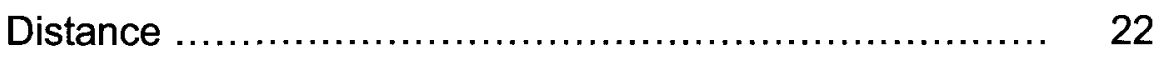

Weight Enumerator ..................................... 23

1.3 PG(2,10)'s Hypothetical Code .............................. 24

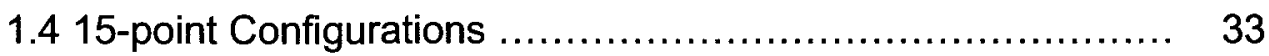

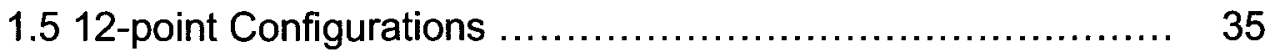

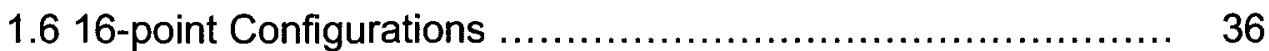




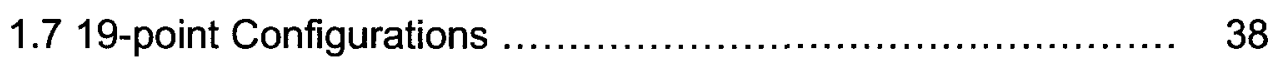

2 Chronology of the Solution 42

2.1 Assmus and Matson ........................................... 42

2.2 MacWilliams, Sloane and Thompson ......................... 43

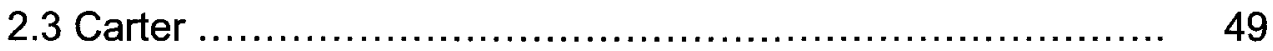

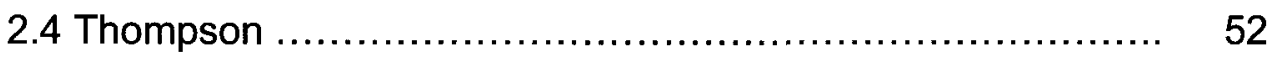

$2.5 \mathrm{Lam}$, Thiel and Swiercz ..................................... 53

3 Implementation $\quad 62$

3.1 C's Weight Enumerator ...................................... 62

3.2 Algorithmic Techniques .................................... 67

3.3 Isomorphism Testing ....................................... 68

3.4 A1 Search .................................................. 72

3.5 A2 Search ..................................................... 75

3.6 Block Search ............................................. 78

3.7 Search Instances ............................................... 85

$M_{15}$ Search ................................................. 85

$\mathbf{M}_{\mathbf{1 6}}$ Search ........................................... 87

Isomorphism Testing Levels ............................... 93

$\boldsymbol{P}_{\mathbf{1 9}}$ Search ............................................... 95 
4 Conclusion 101

$5 \quad$ Further Research 103

$\begin{array}{lll}\text { A Numerical Results of Search } & 105\end{array}$

B Linear System of Weight Enumerator Coefficients 106

$\begin{array}{lll}\text { C } & \text { Row-Reduced Form of Matrix in Appendix B } & 107\end{array}$

$\begin{array}{ll}\text { Bibliography } & 108\end{array}$ 


\section{List of Figures}

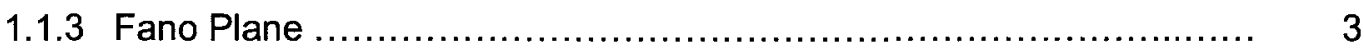

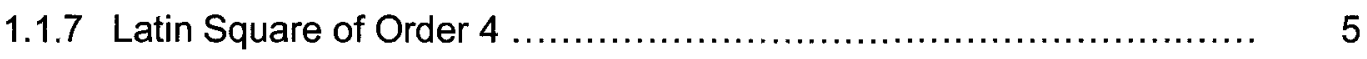

1.1.8 Two Mutually Orthogonal Latin Squares of Order $4 \ldots \ldots \ldots \ldots \ldots \ldots \ldots .6$

1.1.10 Automorphism of the Fano Plane ................................. 8

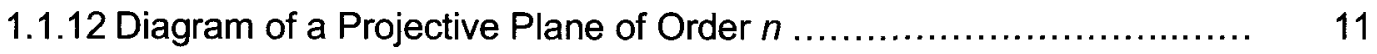

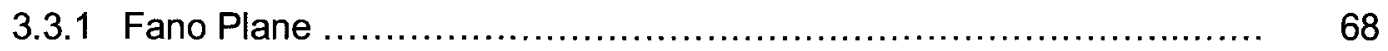

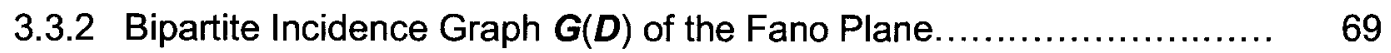




\section{Background}

\subsection{Projective Planes}

1.1.1 Definition (Projective Plane)[2]. A projective plane consists of a set of points, a set of lines and an incidence relation between points and lines with the following properties:

1. Any two distinct points are incident with exactly one line;

2. Any two distinct lines are incident with exactly one point;

3. There exist at least 4 points, with no three of these incident on a line.

Note that property 2 ensures that there are no parallel lines, as opposed to the more "intuitive" Euclidean plane where an alternate axiom is necessary for the existence of parallel lines (i.e. given a line $\ell$ and a point $P$ not incident with $\ell$, there exists exactly one line parallel to $\ell$ which goes through $P$ )[2]. Ironically, for many years mathematicians attempted to remove this explicit axiom by showing that it followed from the other axioms of the Euclidean plane[31]. It took centuries before it was realized that parallel lines need not exist in a given geometry. In fact, multiple parallel lines to a given line may exist through the same point in the Hyperbolic plane [31]. 
We add the following properties to definition 1.1 .1 above to obtain a finite projective plane $\operatorname{PG}(2, n)$, where $n$ denotes the order of the plane [2]:

4. Each line contains exactly $n+1$ points;

5. Property 4 implies that each point is on exactly $n+1$ lines.

1.1.2 Corollary[2]. There exist exactly $n^{2}+n+1$ points and $n^{2}+n+1$ lines in a finite projective plane $\pi$ of order $n$.

Proof. Consider an arbitrary point $P$ of our projective plane $\pi$. By property 5 above, there are $n+1$ lines incident to $P$. By property 4 , since each line contains exactly $n+1$ points, and each of these lines are already incident with the point $P$, each of the lines also contains $n$ other points. So we have $n+1$ lines with $n$ points on each one, and the point $P$, which gives us $n(n+1)+1=n^{2}+n+1$ points. There can be no additional points, since by property 1 this would result in more than $n+1$ lines through point $P$, which would violate property 5 . (Similar reasoning yields $n^{2}+n+1$ lines)

The diagram below depicts the "smallest" finite projective plane, i.e. order 2, also known as the Fano plane [2]: 


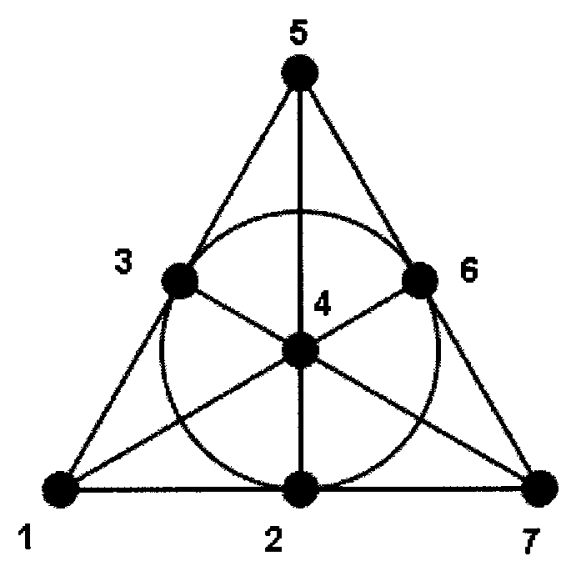

Figure 1.1.3

Note that there are $n^{2}+n+1=7$ points and $n^{2}+n+1=7$ lines, as well as $n+1$ points on every line and every point incident with $n+1$ lines.

Alternatively, a finite projective plane conforms to the definition of a combinatorial design. Specifically:

1.1.4 Definition ( $(v, k, \lambda)$-Balanced Incomplete Block Design)[1]. A design is a pair $(X$,$) where X$ is a set of elements called points, and is a collection of nonempty subsets of $X$ called bocks. $A(v, k, \lambda)-B I B D$ is a design $(X, A)$ which satisfies the following:

1. $|X|=v$

2. each block contains exactly $k$ points

3. every pair of distinct points is contained in exactly $\lambda$ blocks 
A BIBD is symmetric if the number of blocks equals the number of points which implies that every point occurs in exactly $k$ blocks. It is easy to verify that a finite projective plane is a (symmetric) $\left(n^{2}+n+1, n+1,1\right)$-BIBD.

A natural question that arises is "For what orders $n$ can we construct a finite projective plane?". This question ends up being very difficult. On the existence side of things, one can always construct a projective plane of order $q^{s}$ where $q$ is any prime number and $s>0$ [2]. The construction involves using finite fields $[1,2]$. There also exist projective planes of order $q^{s}$ constructed by other methods (the Johnson plane of order 16 for example [10]). However, none of the construction techniques that work for orders $q^{S}$ can be applied to composite numbers which are not a prime power. It is conjectured that finite projective planes exist only for orders $q^{S}[10]$

With regard to non-existence there are only two known results, one of which is the topic of this thesis. The other is the celebrated Bruck-Ryser theorem:

1.1.5 Theorem (Bruck-Ryser)[11]. Suppose that $n$ has either the form $4 k+1$ or $4 k+2$ and that $n$ does not equal the sum of two integral squares. Then $n$ does not occur as the order of a finite projective plane. (we omit the proof, since it is very lengthy) 
This eliminates an infinite number of possible orders for finite projective planes, i.e. $6,14,30, \ldots .$. etc. However it also says nothing about an infinite number of other cases, i.e. $10,12,18,21,22,26, \ldots .$. etc. Nevertheless, it is the only general theorem establishing non-existence since projective planes were "discovered". It should be noted that since R.H. Bruck and H.J. Ryser published their proof in 1949, the theorem was expanded into the Bruck-Ryser-Chowla theorem which encompasses symmetric designs in general [1]. We will not cover it here, since the simpler Bruck-Ryser is enough for the purpose of this thesis.

At this point we present the concept of latin squares since they are related to projective planes.

1.1.6 Definition (Latin square)[1]. A latin square of order $n$ with entries from a set $X$ (often $Z_{n}$ ) where $|X|=n$, is an $n \times n$ array $L$ such every row of $L$ is a permutation of the elements of $\boldsymbol{X}$ and every column of $L$ is a permutation of the elements of $\boldsymbol{X}$.

Figure 1.1.7 below depicts a latin square of order 4 :

$$
\left[\begin{array}{llll}
1 & 2 & 3 & 4 \\
2 & 3 & 4 & 1 \\
3 & 4 & 1 & 2 \\
4 & 1 & 2 & 3
\end{array}\right]
$$

Figure 1.1.7 
1.1.7 Definition (Orthogonal Latin square)[1]. Define a third $n \times n$ array with entries $\left(L_{1}(i, j), L_{2}(i, j)\right)$ from two latin squares $L_{1}$ and $L_{2}$. If in the third array each of the possible $n^{2}$ ordered pairs occur exactly once, $L_{1}$ and $L_{2}$ are orthogonal. Note that two latin squares of the same order are not necessarily orthogonal.

Figure 1.1.8 depicts two orthogonal latin squares of order 4:

$$
L_{1}:=\left[\begin{array}{cccc}
1 & 2 & 3 & 4 \\
4 & 3 & 2 & 1 \\
2 & 1 & 4 & 3 \\
3 & 4 & 1 & 2
\end{array}\right] \quad L_{2}:=\left[\begin{array}{cccc}
1 & 2 & 3 & 4 \\
3 & 4 & 1 & 2 \\
4 & 3 & 2 & 1 \\
2 & 1 & 4 & 3
\end{array}\right]
$$

Figure 1.1.8

$$
\left[\begin{array}{l}
(1,1)(2,2)(3,3)(4,4) \\
(4,3)(3,4)(2,1)(1,2) \\
(2,4)(1,3)(4,2)(3,1) \\
(3,2)(4,1)(1,4)(2,3)
\end{array}\right]
$$

In a set of latin squares of order $n$, if all latin squares are pair-wise orthogonal then they are mutually orthogonal [1]. We denote a set of $r$ mutually orthogonal latin squares of order $n$ as $r \operatorname{MOLS}(n)$. An important question that arises is, "What is the maximum number, $r$, of $\operatorname{MOLS}(n)$ ?", traditionally denoted as $N(n) . N(n)$ attains a maximum value of $n-1$ when $n$ is a prime power, and the 
existence/non-existence of $n-1 \mathrm{MOLS}(n)$ is equivalent to the existence/nonexistence of a projective plane of order $n$ (see [1] for a proof of these statements). Euler conjectured that for latin squares of order $n$ where $n$ is $2 \bmod (4), N(n)=1$ [12]. This is trivially true for order 2 , and was proven true for order 6 (note that Bruck-Ryser rules out order 6 for a projective plane) [13]. Had Euler's conjecture actually been true, then order 10 would have been ruled out as the order of a projective plane. However, a pair of mutually orthogonal latin squares of order 10 was first discovered in 1959, disproving Euler's conjecture [14]. Note that 3 MOLS(10) have never been discovered, nor disproved. But based on Lam's proof of the non-existence of a projective plane of order 10 , we know that $N(10)<$ 9. In addition Dow has shown that $N(n)<n-1$ implies $N(n)<n-2[38,39]$. Hence, $2 \leq N(10)<8$.

Another important concept related to planes is that of automorphism. The traditional term for automorphism of projective planes is collineation.

1.1.9 Definition (Collineation)[2]. A collineation is a point-to-point and line-toline transformation from the plane to itself, that preserves the relation of incidence.

In other words when we map (i.e. transform) points to other points, the end result preserves the incidence of the original structure, i.e. collinear points remain 
collinear. As an example when we apply the following mapping of points from the Fano plane to itself, we obtain an automorphism since incidence is preserved.

$$
1 \rightarrow 1,2 \rightarrow 3,3 \rightarrow 2,4 \rightarrow 4,5 \rightarrow 7,6 \rightarrow 6,7 \rightarrow 5
$$
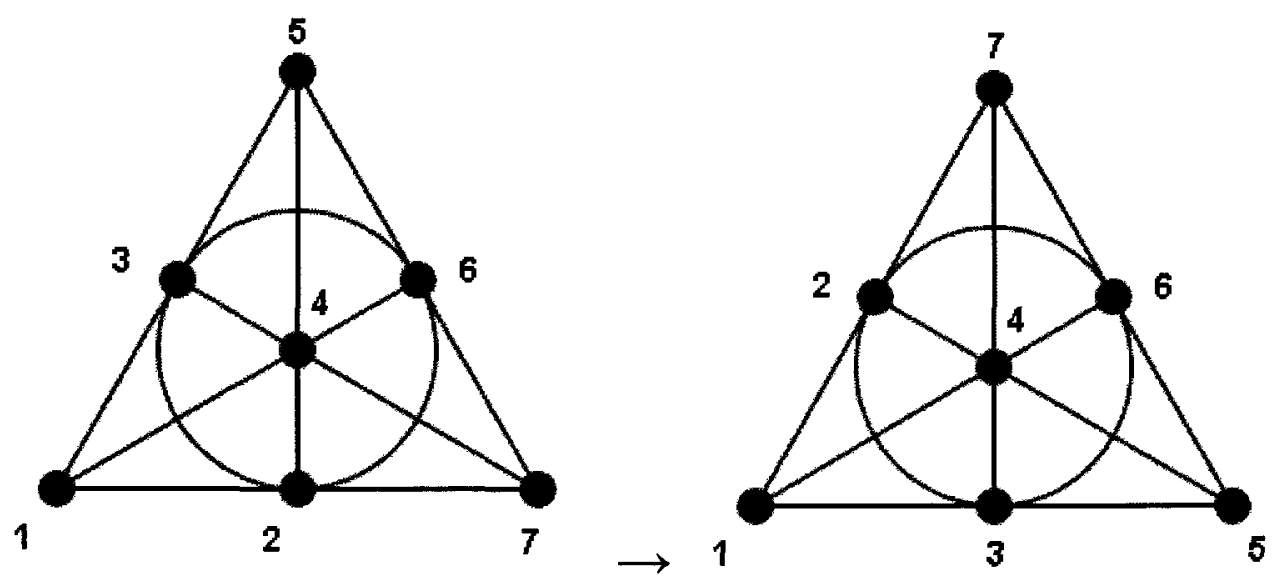

Figure 1.1.10

A series of papers written in the 1970 s and 1980 s showed that a projective plane of order 10 would have no automorphisms other than a trivial mapping of all points to themselves $[15,16,17,18]$. Not only is this unusual for a projective plane (all known planes have symmetry), but it also makes the task of finding one even more difficult. However, we will see how isomorphism (i.e. automorphism of partial projective planes) is a necessary tool to solve the main problem of this thesis. 
A convenient way to represent a projective plane of finite order is by its incidence matrix [1]. Specifically, for a projective plane of order $n$, we define an $n^{2}+n+1$ by $n^{2}+n+1$ binary matrix $\boldsymbol{A}$ where the rows represent the lines and the columns represent the points. If a line $i$ is incident with a point $j$, then the entry $a_{l j}$ is 1 , otherwise the entry is 0 . For example, the figure below is the incidence matrix of a projective plane of order 2 (specifically the Fano plane depicted in figure 1).

\begin{tabular}{c|lllllll|}
\multicolumn{1}{c}{} & P1 & P2 & P3 & P4 & P5 & P6 & P7 \\
\cline { 2 - 8 } L1 & 1 & 1 & 0 & 0 & 0 & 0 & 1 \\
L2 & 0 & 0 & 1 & 1 & 0 & 0 & 1 \\
L3 & 0 & 0 & 0 & 0 & 1 & 1 & 1 \\
L4 & 1 & 0 & 1 & 0 & 1 & 0 & 0 \\
L5 & 1 & 0 & 0 & 1 & 0 & 1 & 0 \\
L6 & 0 & 1 & 1 & 0 & 0 & 1 & 0 \\
L7 & 0 & 1 & 0 & 1 & 1 & 0 & 0 \\
\cline { 2 - 8 } & & & & & & &
\end{tabular}

We can now proceed to evaluate how difficult a task it is to attempt to "find" a projective plane of order 10. Can we search for 9 MOLS(10) to find a projective plane of order $10 ?$ There exist approximately $3.5 \times 10^{16}$ main class ${ }^{\Psi}$ latin squares of order 10 which yields $10^{147} 9$-subsets, obviously too large a search space.

A more reasonable way might be to search for its associated incidence matrix, given the absence of any other method. However such a matrix would have 111

\footnotetext{
$\Psi$ "Isomorphic" latın squares
} 
columns and 111 rows with eleven 1 's in each row and each column, and 0's everywhere else. There are approximately $10^{1629}$ such matrices. Of these, we would need to find one in which the inner product of every pair of rows and every pair of columns is 1 . Assuming (very optimistically) that a computer can generate and verify $10^{10}$ matrices per second, it would take 12 billion computers $10^{1592}$ times longer than the estimated current age of the universe [40] to verify that no such matrix satisfies the given conditions (we would be better off searching for 9 MOLS(10)). However, one need not verify the inner product just at the end of the matrix construction: indeed this can be checked as rows are added to the matrix. This is traditionally known as a backtracking algorithm [6]. For example suppose, as in the example below, that we have two consistent rows (inner product is 1 ) for a projective plane of order 2 , and that we wish to continue building it.

\begin{tabular}{|c|c|c|c|c|c|c|c|}
\hline & P1 & P2 & P3 & P4 & P5 & P6 & P7 \\
\hline L.1 & 1 & 1 & 0 & 0 & 0 & 0 & 1 \\
\hline L2 & 0 & 0 & 1 & 1 & 0 & 0 & 1 \\
\hline
\end{tabular}

Then the consistent choices for a third row are 0000111, 1010100, 0101010, etc... We would discard 1110000 for example, since its inner product with row 1 is 2 . We would then proceed with the $4^{\text {th }}$ row, and consider only choices that are consistent with the previous three rows. We proceed until we have constructed a matrix or no choices are available at the current level, in which case we "backtrack" to the next choice at the previous level. This is in essence the method used to prove the non-existence of a projective plane of order 10 , 
although we shall see that it can only be accomplished with massive reductions to the available choices at each level of the search tree.

One way to reduce choices at each level of the construction is to use isomorphism as discussed earlier. In fact for a projective plane of order 10, it is possible to specify the first 22 rows of the incidence matrix in a unique way, up to isomorphism.

1.1.11 Theorem. For a plane of order $n$, we can uniquely determine the first $(n+1)^{*} 2$ rows of its incidence matrix (i.e. up to isomorphism, there is only one instance of these rows).

Proof. Consider the diagram in figure 1.1.12:

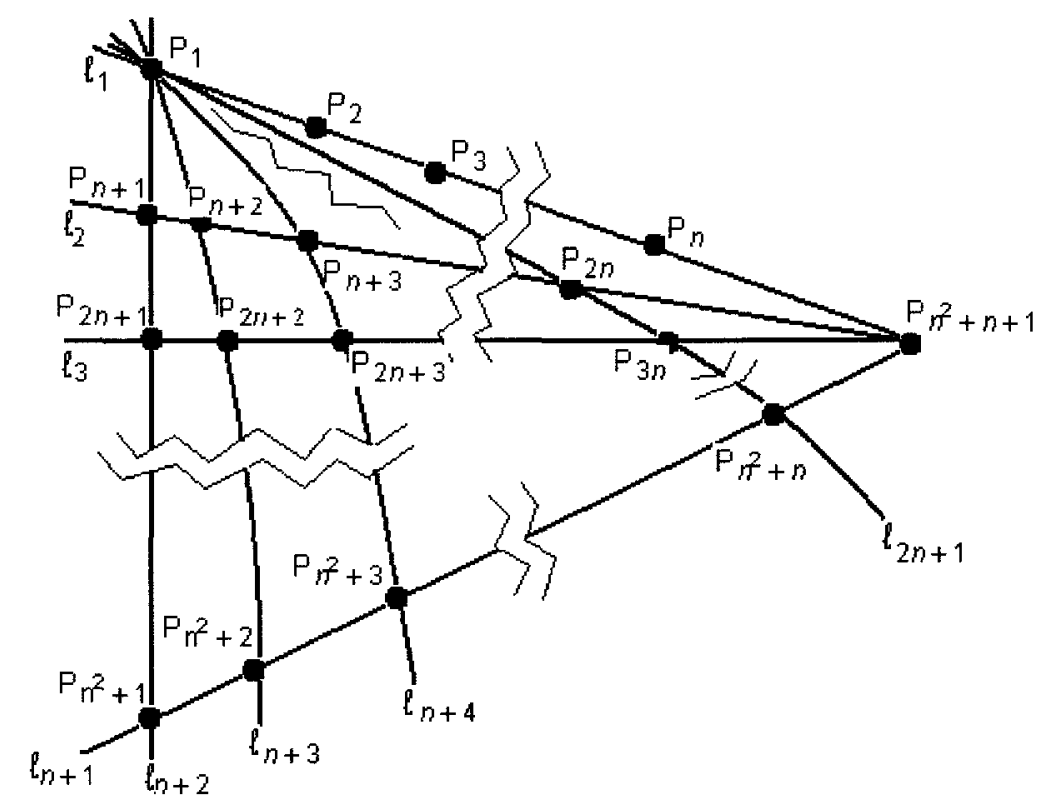

Figure 1.1.12 
We know that there is at least one point on the plane (labelled $\mathrm{P}_{n}^{2}+n+1$ above). It has exactly $n+1$ lines going through it. Each of these lines has exactly $n+1$ unique points on it (including our original point). Hence we have all of our $n^{*}(n+1)+1=n^{2}+n+1$ points and $n+1$ of our lines. And without loss of generality we can name, as we wish, the lines $\ell_{1}, \ell_{2}, \ldots, \ell_{n}+1$ and the points on those lines, as above. This accounts for the first $n+1$ lines. To more easily illustrate based on the diagram above, here are the first $n+1$ lines of the matrix of a projective plane of order 4 as per the diagram:

$\begin{array}{lllllllllllllllllllllll}\mathrm{P} & \\ \mathrm{L} & 1 & 2 & 3 & 4 & 5 & 6 & 7 & 8 & 9 & 1 & 1 & 1 & 1 & 1 & 1 & 1 & 1 & 1 & 1 & 2 & 2 \\ \mathrm{~L} \\ 1\end{array}$

Next we know that $n$ more lines must go through $\mathrm{P}_{1}$, and each must intersect all existing lines in exactly one point. We can set the first of these lines, $l_{n}+2$, to be incident with the points $P_{n+1}, P_{2 n+1}, \ldots, P_{n \times n+1}$, the second of these lines, $\ell_{n+3}$, with the points $P_{n+2}, P_{2 n+2}, \ldots, P_{n \times n+2}, \ldots$, and the $n^{\text {th }}$ line, $\mathrm{l}_{2 n+1}$, with $\mathrm{P}_{n+n}, \mathrm{P}_{2 n+n}, \ldots, \mathrm{P}_{n \times n+n}$. As an aid to illustrate, consider the 4 (italicized) rows added to our matrix of a projective plane of order 4 : 


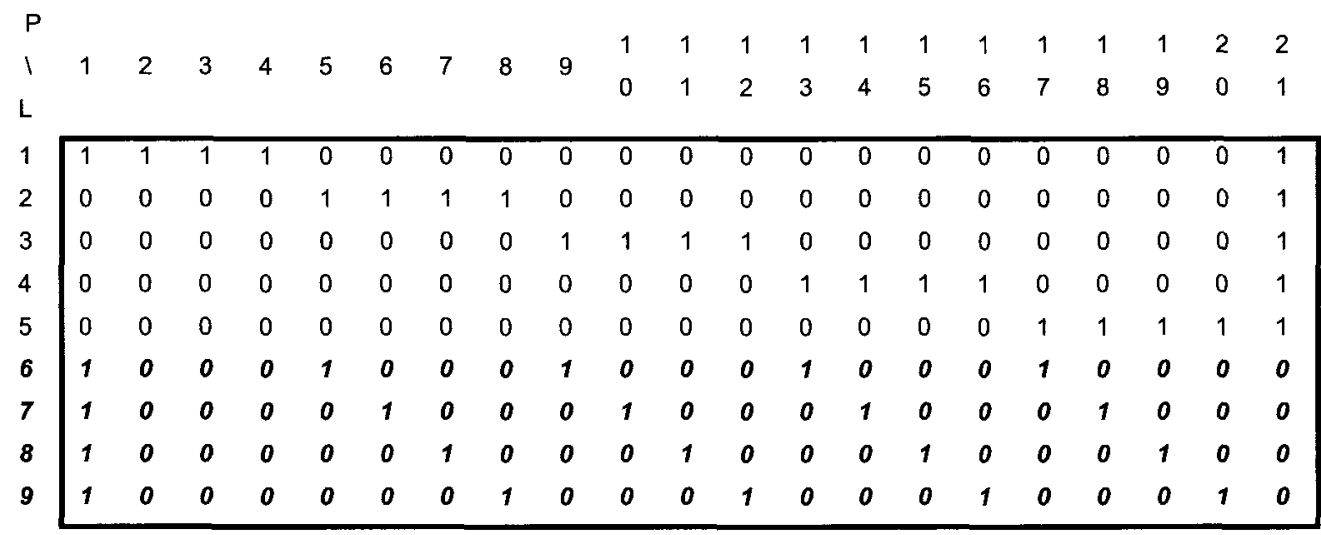

Note, we could have chosen any other points than $\mathrm{P}_{4 i+1}$ (where $i=1$ to 4 ) for $\ell_{6}$ within the groups $5-8,9-12,13-16$, and $17-20$. However it is possible to map the points of any instance of this row without changing the rows 1 through 5 . For example, suppose $\ell_{6}$ (row 6) were incident with $P_{6}, P_{11}, P_{15}$, and $P_{18}$. Then we simply apply the mapping 6->5, 5->6, 11->9, 9->11, 15->13, 13->15, 18->17, 17->18. This would produce the row 6 as in the above matrix, and preserve the rows 1 to 5 : because the mappings apply within the groups $5-8,9-12,13-16$, and 17-20, the first 5 lines remain unchanged (try it!). The same applies to $\ell_{7}$ : it can have any permutation of points, other than the $\mathrm{P}_{4 i+1}$ (where $i=1$ to 4 ) since it is already incident with $\ell_{6}$ through point $P_{1}$. Hence to transform a given $\ell_{7}$, the mapping applies within groups 6-8, 10-12, 14-16 and 18-20, and no such mapping will alter any of the previous rows in the incidence matrix. This applies to the two additional lines as well ( $\ell_{8}$ and $\ell_{9}$ ). And it applies in the same way in the general case of $n$. How does one obtain the additional line $\ell(n+1) \times 2$ ? We 
go back to our matrix of the projective plane of order 4 , and examine the additional (italicized) line below:

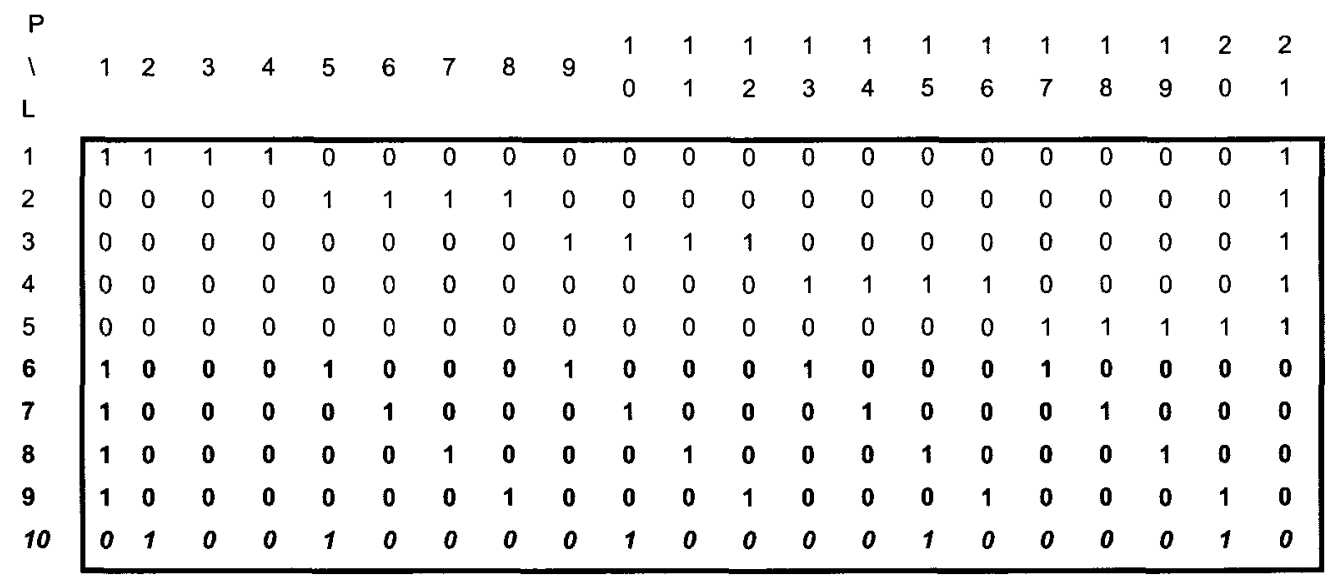

Consider the groups of columns $1-4,5-8,9-12,13-16$ and $17-20 . \ell_{10}$ must be incident with exactly one point in each of these groups. For 1-4, we cannot choose $\mathrm{P}_{1}$ since it is already incident with 5 lines. For the other groups, any combination is valid except the following: we cannot choose any two points whose index mod 4 is equal, since this is accounted for in the previous 4 lines. In other words, we must come up with four points that have different mod 4 values, i.e. a permutation on the set $\{0,1,2,3\}$. It turns out that the choice above happens to be unique up to isomorphism ${ }^{\Psi}$.

We can further extend the unique matrix by adding the following columns (italicized):

\footnotetext{
${ }^{\Psi}$ This fact has been verified with computational methods described later on
} 


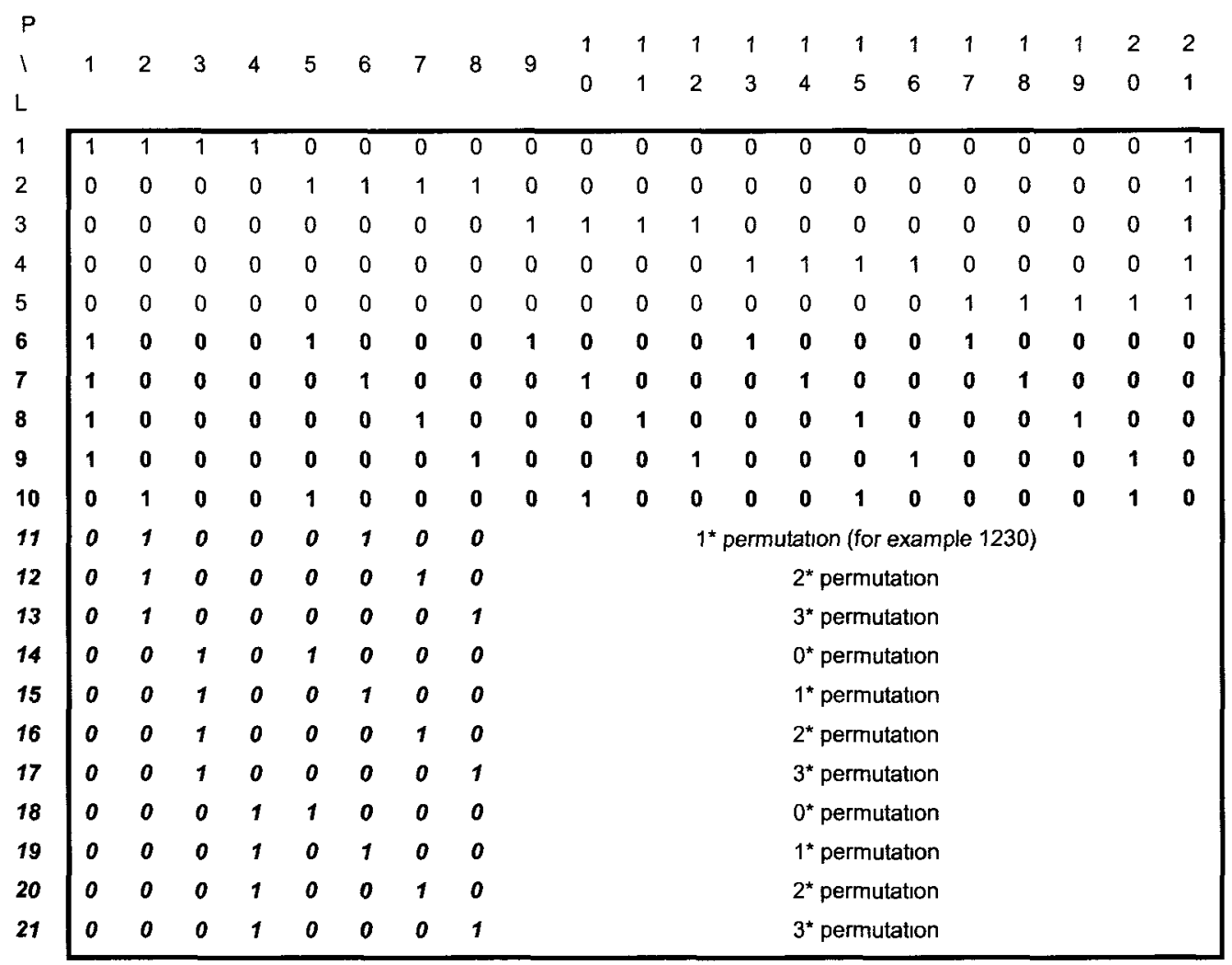

The first four columns account for the additional lines that must go through points $P_{2}, P_{3}$ and $P_{4}$. Then columns 5 through 8 account for the fact that each of $\left\{P_{2}\right.$, $\left.P_{3}, P_{4}\right\}$ must be collinear with every instance of $\left\{P_{5}, P_{6}, P_{7}, P_{8}\right\}$ on a unique line. Any more additions to the matrix will no longer necessarily be unique up to isomorphism.

Now using the permutation idea to fill out the rest of the matrix, we are looking to find permutations on $\{0,1,2,3\}$ in the following manner: 
- The first permutation is 0123 and is free. It maps to line 10 (1st point in group $5-8,2^{\text {nd }}$ in group $9-12,3^{\text {rd }}$ in group $13-16,4^{\text {th }}$ in group $17-20$ );

- We need 2 more permutations that begin with 0 and map to lines 14 and 18 ;

- We need 3 permutations that begin with 1 and map to lines 11,15 , and 19 ;

- We need 3 permutations that begin with 2 and map to lines 12,16 , and 20 ;

- We need 3 permutations that begin with 3 and map to lines 13,17 , and 21;

- Permutations must not violate the axioms of a projective plane, i.e. two points cannot occur together on more than one line.

The last statement above is where the difficulty really lies. First off, how can we identify that two permutations are not compatible, i.e. that they would violate the integrity of the plane? This is best illustrated by examining permutations themselves. Consider 0123 and 2103: this yields the partial line incident with points $P_{5}, P_{10}, P_{15}, P_{20}$ and the partial line incident with points $P_{7}, P_{10}, P_{13}, P_{20}$. These partial lines both contain $P_{10}$ and $P_{20}$, so the permutations that yield them are not compatible. In general terms, permutations are incompatible if a pair of identical moduli occur at the same indexes on the permutations, i.e. $0123-2103$.

Unfortunately while it is easy to determine if permutations are compatible, it is very difficult to find an efficient method to construct a set of compatible 
permutations, short of exhaustive search which runs in exponential time. However, we can use the idea to analyze how difficult it is to construct a plane of order 10: we are looking at permutations on $\{0,1,2,3,4,5,6,7,8,9\}$, and the first permutation is 0123456789 . So how many permutations compatible to 0123456789 exist? Let's just consider the ones that start with 0 , since we need to find 8 more of these. A permutation that begins with 0 and is compatible with 0123456789 must have derangement on all positions other than the first [19]. For example, all $01^{*}, 0 ? 2^{*}, 0 ? ? 3^{*}$, etc permutations are incompatible. There happen to be 133496 derangements on permutations of 9 symbols [19]. We are interested in obtaining one of each of permutations beginning with $02,03,04,05$ $06,07,08$, and 09 . For $02^{*}$ permutations, there are $133496 / 8=16687$ possibilities (i.e. the total number of derangements is divided into 8 partitions [19]). Next we choose a permutation starting with 03 . There are not 16687 of these as with the $02^{*}$ permutations, because the chosen $02^{*}$ permutation further limits the available $03^{*}$ permutations in the same manner as the initial 0123456789 permutation limits all subsequent permutations. It is however more difficult to derive the number of compatible $03^{*}$ permutations, which is best left to a computer program. Furthermore the actual number depends on the specific choice of the $02^{*}$ permutation. The same applies in order to derive compatible $04^{\star}$ permutations, 05* permutations, and so on up to $09^{*}$ permutations. The table below shows the order of magnitude for compatible $0^{*}$ permutations: 


\begin{tabular}{|c|c|}
\hline $02^{*}$ & 16,687 \\
\hline $03^{\star}$ & $10^{4}$ \\
\hline $04^{*}$ & $10^{3}$ \\
\hline $05^{*}$ & $10^{3}$ \\
\hline $06^{*}$ & $10^{2}$ \\
\hline $07^{\star}$ & $10^{1}$ \\
\hline $08^{*}$ & $10^{1}$ \\
\hline $09^{\star}$ & $10^{1}$ \\
\hline
\end{tabular}

Following the derivation of the $0^{*}$ permutations, we would then move on to the $1^{*}$ permutations, $2^{*}$ permutations, etc... Remember that the $0^{*}$ permutations restrict all following permutations $\left(1^{*}, 2^{*}, \ldots, 9^{*}\right)$. Once again using a computer program we can calculate the orders of magnitude for the compatible $1^{*}$ permutations, which are noted in the following table:

\begin{tabular}{|c|c|}
\hline $10^{\star}$ & $10^{3}$ \\
\hline $12^{\star}$ & $10^{3}$ \\
\hline $13^{\star}$ & $10^{2}$ \\
\hline $14^{\star}$ & $10^{2}$ \\
\hline $15^{\star}$ & $10^{1}$ \\
\hline $16^{\star}$ & $10^{1}$ \\
\hline $17^{\star}$ & $10^{1}$ \\
\hline $18^{\star}$ & $10^{0}$ \\
\hline $19^{\star}$ & $10^{0}$ \\
\hline
\end{tabular}


In order to generate a full projective plane of order 10 , we would continue on to find $2^{*}, 3^{*}, \ldots, 9^{*}$ compatible permutation levels. Each level would be further restricted by previous permutations. However, calculating the orders of magnitude for subsequent levels requires too much $\mathrm{CPU}$ time (since it is the same as actually finding a plane). We do know that a current level (say $3^{\star}$ ) has fewer compatible choices than a previous level (say $2^{*}$ ). But let's assume conservatively that all $2^{*}, 3^{*}, \ldots, 9^{*}$ levels have the same orders of magnitude as the $1^{*}$ level, we then obtain a rough upper bound of $10^{136}$. While this is a gross overestimation, it is still a smaller search space than searching for 9 MOLS(10) $\left(10^{147}\right)$, or generating and verifying all $10^{1629}$ possible incidence matrices with 111 columns and 111 rows with eleven 1's in each row and each column, and 0's everywhere else. However it is still much too large a search space. By using Knuth's method of estimating the size of a search tree [20], we obtain on the search space a tighter ${ }^{\Psi}$ bound of $10^{68}$, but which is still too large for a computer to handle.

Note that the manner in which we build the plane with compatible permutations is actually a backtrack search as described earlier. If we combine this technique with isomorphism, we can reduce the search space further. In other words, not all 16,687 compatible $02^{*}$ permutations are unique up to isomorphism. In fact there are only 22 unique compatible $02^{*}$ permutations among the 16,687

\footnotetext{
${ }^{\Psi}$ This value is the average of 10,000 runs, which ranged from $10^{39}$ to $10^{84}$.
} 
possibilities ${ }^{\Psi}$, and unique compatible $03^{\star}$ permutations are limited to $10^{3}$ rather than $10^{4}$. Unfortunately the benefits diminish after only a few levels, so backtracking along with isomorphism are not enough to reduce the search tree to a manageable level. Once again by using Knuth's method, the estimated search space when backtracking and isomorphism are combined is $10^{26}$. Although this is much smaller than the previous methods, it is still too large. We need a way to reduce the space further.

\footnotetext{
${ }^{\Psi}$ The programming techniques used to eliminate isomorphs will be introduced later on
} 


\subsection{Error Correcting Codes}

What are error correcting codes? Consider the problem of sending a message over a noisy channel, for example downloading data over a phone line. Specifically, suppose the message sent consists of the bit string 110011: a noisy channel may cause the message received to be 110010 . The goal of a binary error-correcting code is to detect and hopefully correct errors that may have occurred during transmission, which is accomplished by adjoining redundant bits to the original string [21].

In designing an error-correcting code one needs to consider all possible messages that can be transmitted. Once this "message space" is properly identified, one must determine the desired maximum number of errors that can occur yet still be corrected. For example, consider the simple message space consisting of the bit strings $00,01,10$, and 11 . One possible coding technique is simply to triple each message, i.e. $00 \rightarrow 000000,01 \rightarrow 010101,10 \rightarrow 101010$, $11 \rightarrow 111111$, and transmit the equivalent encoded string in lieu of the original string.

All encoded strings comprise the list of codewords in our error-correcting code. Now suppose we want to transmit the message 01: we send 010101, but it may happen due to noise on the channel that the message received is 110101 . The receiver would look at the list of possible correct messages and determine that an 
error has occurred. A comparison would yield that the valid message most similar to the received message is 010101 , which corresponds to the original message.

A more complete analysis of all single bit errors in the above list of codewords confirms that by performing a comparison with all valid codewords and retaining the most similar we always obtain the correct original codeword. This comparison is formally referred to as finding the codeword that has the smallest distance ${ }^{1}$ from the received message. The number of errors within a message that a code will successfully be able to correct depends on the minimum distance between its codewords. The code above, for instance, would not be able to successfully detect 2 errors in a given message, since a code can only detect and correct ( $d$ 1)/2 errors where $d=3$ is the smallest distance among all of the codewords.

Since all of the codewords above have distance 3 from each other, the code will indeed be capable of detecting and correcting only 1 error. Formally our code is a $[6,2]$ Hamming code with minimum distance 3 , meaning that its codewords are 6 digits of which 2 digits are the original message [21].

It is advantageous to derive our codes from algebraic structures. In particular, binary linear error-correcting codes are defined over the finite field $\mathbf{F}_{\mathbf{2}}$, and the codewords are elements from the vector subspace $\mathbf{F}_{2}{ }^{\mathbf{n}}$ [21]. Codes defined in

\footnotetext{
${ }^{1}$ The distance between two bit-strings is the number of positions in which they differ
} 
the latter way are efficient in terms of maximizing error correction and minimizing redundancy. We can define a binary linear code by specifying its generator matrix: the codewords are then linear combinations of the rows of the matrix.

For the purposes of this thesis, we need to discuss another property of the individual codewords, specifically its weight which is simply the number of 1's it contains. For example, the weight of 010101 is 3 . The notation $w_{i}$ refers to the number of codewords with weight $i$ in a given code. The following expression, called the weight enumerator of a code, is a polynomial which relates the weights of its codewords $[9,21]$ :

$$
\sum_{i=0}^{\text {length }} w_{i} x^{i}
$$

where $w_{i}$ is the number of codewords of weight $i$. 


\subsection{PG(2,10)'s Hypothetical Code}

The first crucial step that eventually led to a solution of the existence of a projective plane of order 10 was a talk that E.F. Assmus presented at the conference on "Combinatorial Aspects of Finite Geometries" in Oberwolfach Germany in April 1970. In particular, Assmus suggested that the analysis of the binary error-correcting code associated to the hypothetical incidence matrix of a projective plane of order 10 would yield some extremely important information. Specifically, that binary error-correcting code $\boldsymbol{C}$ is the vector space over $\mathbf{F}_{2}{ }^{\mathbf{n}}$ generated by the rows of the incidence matrix $\boldsymbol{A}$ of a projective plane of order 10 . Many sources contain an analysis of the resultant error-correcting code $C$, with Assmus and Mattson leading the way $[4,5,22]$. We will note the important results here for the sake of completeness.

1.3.1 Lemma. The dimension of $C$ is 56 .

Proof. i) $\operatorname{dim} C \geq 56$.

For a projective plane of order $n, \boldsymbol{A} \boldsymbol{A}^{\boldsymbol{t}}=n \mathbf{I}+\mathbf{J}^{\Psi}$. Thus, $\operatorname{det} \boldsymbol{A}=n^{\left.\left(n^{2}+n\right) / 2\right)}(n+1)$.

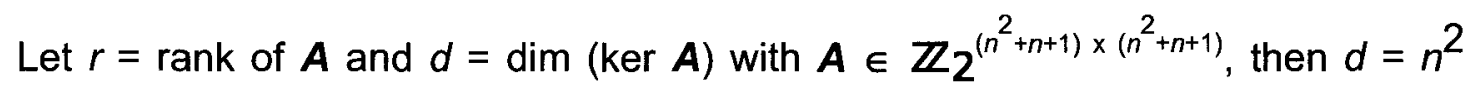
$+n+1-r$. Let $\left\{a_{1}, a_{2}, \ldots, a_{d}\right\}$ be row vectors which span the kernel of $\boldsymbol{A}$.

\footnotetext{
${ }^{\Psi} \mathbf{I}$ and $\mathbf{J}$ are the identity and all $1 \mathrm{~s}$ matnces, respectively
} 
Furthermore, arrange these so that for each $a_{j}$, a ' 1 ' occurs in the $i^{\text {th }}$ position with all zeros before ${ }^{\Psi}$. We can then define a matrix $B$ :

$$
B:=\left[\begin{array}{c|c}
\begin{array}{c}
a_{1} \\
a_{2} \\
a_{d} \\
a_{d r}
\end{array} \\
\hline o_{r} & I_{r}
\end{array}\right]
$$

$B$ is upper triangular with diagonal 1's and has det $=1$. Hence $\operatorname{det} \mathbf{B A}=n^{\left(\left(n^{2}+n\right) / 2\right)}(n+1)$. In addition, the first $d$ rows of $\boldsymbol{B A}$ 's entries are multiples of 2 , so $2^{d} \mid \operatorname{det} B \boldsymbol{B A}$. So for $n=10, d \leq 55$ and $r \geq 56$. Hence $\operatorname{dim} \boldsymbol{C} \geq 56$.

ii) $\operatorname{dim} C \leq 56$.

Let $C^{\bullet}$ denote the code of length 112 which we obtain by adjoining a parity bit to $C$, so that all codewords of $C^{\bullet}$ have even weight. Let $C^{\bullet \perp}$ denote the orthogonal complement of $C^{\bullet}$ in $\mathbf{F}_{\mathbf{2}}{ }^{\mathbf{1 1 2}}$. The rows of $\boldsymbol{A}$ in $C^{\bullet}$ all have weight 12 and intersect pairwise in two points. Therefore the generators of $c^{\bullet}$ are all contained in $c^{\bullet} \perp$, so $C^{\bullet} \subseteq C^{\bullet \perp}$ and $\operatorname{dim} C^{\bullet} \leq 112 / 2=56$. Since $\operatorname{dim} C^{\bullet}$ must also be $\geq C$, we have that $\operatorname{dim} C \leq 56$.

\footnotetext{
${ }^{\Psi}$ This can be done because the vectors span a subspace over $\mathbb{Z}_{2}$, i.e. they are already in a row-echelon form (at most requiring row and/or column permutations).
} 
1.3.2 Lemma. All codewords in $C^{\bullet}$ are congruent to 0 (mod 4), and all codewords in $\mathbf{C}$ are congruent to 0 or $3(\bmod 4)$.

Proof.

i) All generators of $C^{\bullet}$ have weight 12 by definition.

ii ) Let $v, w$ be any two codewords in $C^{\bullet}$. Consider the following expression ${ }^{\Psi}$ :

$$
|v+w|=|v|+|w|-2|v \cap w|
$$

Since $C^{\bullet} \subseteq C^{\bullet \perp},|v \cap w|$ must be even. And thus each individual term in the expression must also be divisible by 4 .

iii ) By definition, any codeword has in $\boldsymbol{C}$ has weight equal to or 1 less than its associated codeword in $\mathbf{C}^{\bullet}$.

1.3.3 Lemma. For $\ell$ any line of the plane and $v$ any codeword in $c,|\ell \cap v|$ is congruent to $|v|$ (mod 2), i.e. an even weight codeword intersects any line of the plane in an even number of points and an odd weight codeword intersects any line of the plane in an odd number of points.

\footnotetext{
${ }^{\Psi}$ Throughout this thesis, codewords and lines are treated interchangeably as both vectors and sets. The indexes of the 1-bits on the vectors correspond to membership in the sets.
} 
Proof. Since $C^{\bullet} \subseteq C^{\bullet} \perp,\left|\ell^{\bullet} \cap v^{\bullet}\right|$ is even (where $\ell^{\bullet}$ and $v^{\bullet}$ are simply $\ell$ and $v^{\prime} s$ associated line and codeword in $C^{\bullet}$ respectively).

i) If $v$ has even weight, then $|\ell \cap v|=\left|\ell^{\bullet} \cap v^{\bullet}\right| \equiv 0(\bmod 2)$.

ii) If $v$ has odd weight, then $|\ell \cap v|=\left|\ell^{\bullet} \cap v^{\bullet}\right|-1 \equiv 0(\bmod 2)$.

1.3.4 Lemma. The minimum weight of a (non-zero) codeword in $\boldsymbol{C}$ is 11 .

Proof.

i) If codeword $v$ has even (non-zero) weight and point $P \in v$ (we can think of each $v \in \boldsymbol{C}$ as an incidence vector of points since $v$ is nothing more than a sum of lines). By the properties of $\mathrm{PG}(2,10), 11$ lines are incident with $P$ and by lemma 1.3.3 $v$ must intersect each of these lines in another point (one for each line). Hence $|v| \geq 12$

ii ) If $v$ has odd weight and $P \notin v$. By the properties of $P G(2,10), 11$ lines are incident with $P$ and by lemma 1.3.3 $\mathrm{v}$ must intersect each of these lines in at least one point (one for each line). Hence $|v| \geq 11$.

1.3.5 Lemma. The codewords of weight 11 in $\mathbf{C}$ are exactly the 111 lines of the plane. 
Proof.

i) By definition all lines are in $C$, since they are the generators of the code.

ii) Suppose that $|v|=11$. Let $\ell$ be a line through $P, Q \in v$. There must be a third point of $v$ on $\ell$, since an odd weight codeword will intersect a line in an odd number of points. If $v \neq \ell$, then there exists a point $x \in \ell \mid v$. The 10 other lines through $x$ must intersect $v$ in at least one point (an odd number). But this implies $|v| \geq 13$, contradicting the fact that $|v|=11$. Hence $v$ must equal $\ell$.

1.3.6 Lemma. The codewords of weight 12 in C are precisely the hyperovals ${ }^{\Psi}$ (sets of 12 points where no three are collinear) of the plane.

Proof.

i) Let $v \in C$ and $|v|=12$. Consider a line $\ell$ : by lemma 1.3.3, it must intersect $v$ in an even number of points, say $2 s$. Take one of these points: there are 11 lines which go through it (including $\ell$ ), and each must distinctly go through at least one more point of $v$. Hence $2 s+10 \leq 12$, implying $2 s=0$ or 2 . Therefore, $v$ is a set of 12 points of which no 3 are collinear, i.e. $v$ is a hyperoval.

ii ) Let $\sigma$ be a hyperoval in the plane, with 12 points. Consider the set of lines through the points of $\sigma$ (each line intersecting $\sigma$ in 0 or 2 points). There are 66

\footnotetext{
${ }^{\Psi}$ All previous sources on the existence of $\mathrm{PG}(2,10)$ refer to, a set of 12 points where no three are collinear, as an oval However an oval in PG $(2,10)$ is in fact, a set of 11 points where no three are collinear Therefore, we will use the proper designation for a set of 12 points where no three are collinear, $\imath e$ hyperoval, in this thesis
} 
lines through the pairs of points of $\sigma$ (11 lines through each point of $\sigma)$, and 45 lines that do not intersect $\sigma$. If we take the vector sum of the 66 lines over $\mathbf{F}_{\mathbf{2}}$, we will obtain $11(\bmod 2)=1$ for each point in $\sigma$. The other 99 points not in $\sigma$ each appear on 6 of the 66 lines through $\sigma$, i.e. $6(\bmod 2)=0$ for each point not in $\sigma$. Therefore the vector sum (a codeword in C) of these 66 lines is precisely the points of $\sigma$.

We now tackle C's weight enumerator. As mentioned briefly in the last section, it is a polynomial which relates the weights of its codewords:

$$
\sum_{i=0}^{\text {length }} w_{i} x^{i}
$$

Alternatively, we can represent the enumerator as $\boldsymbol{W}_{C}(x, y)$. For a code of length $n$, this is an $n^{\text {th }}$ degree polynomial homogeneous in the variables $x$ and $y$, with a coefficient $\mathrm{A}_{i}$ of $x^{n-i} y^{j}$ equaling the number of codewords of weight $i$. We already have some information regarding $W_{C}(x, y)$ from the preceding lemmas:

- $\mathrm{A}_{0}=1 ;$

- $A_{1}=A_{2}=\ldots=A_{10}=0$;

- $A_{11}=111$;

- $A_{i}=0$ when $/$ is congruent to $1,2(\bmod 4)$, unknown otherwise;

- $\mathrm{A}_{i}=\mathrm{A}_{111-i}$, since the sum of all lines is the all-one codeword. 
We can obtain even more information by appealing to the MacWilliams identities for linear codes:

1.3.7 Theorem (MacWilliams Identity)[21]. If $\boldsymbol{C}$ is an [n, $k]$ binary linear code with dual code $\mathrm{C}^{\perp}$, then,

$$
2^{k} W_{C}(x, y)=W_{C}^{\perp}(x+y, x-y)
$$

(we do not present the proof since it is lengthy)

As previously indicated, $\boldsymbol{C}$ is a $[111,56]$ binary linear code, and it can be shown that its orthogonal complement $\boldsymbol{C}^{\perp}$ is simply the codewords of $\boldsymbol{C}$ which have even weight ${ }^{1}$. Hence the left hand side of the identity is the terms of the right hand side of even degree applied to the variables $(x+y)$ and $(x-y)$. The coefficients (the $A_{j}$ ) of $C$ 's weight enumerator can be determined by using the equality obtained due to the MacWilliams identity to extract a system of equations expressed in terms of the $A_{i}$. Solving ${ }^{2}$ this system yields a space of dimension 3 , i.e. a fixed set of 3 weights determines all other weights. The relation of particular interest to us is:

$$
w_{19}=24675+141 w_{12}-27 w_{15}+7 w_{16}
$$

It is easier computationally to deal with coefficients of lesser weights $\left(\boldsymbol{w}_{12}, w_{15}\right.$, $w_{16}$, and $w_{19}$ are the least weights in the code). In particular $w_{19}$ is uniquely

\footnotetext{
${ }^{1}$ This statement is elaborated in the implementation section

${ }^{2}$ A complete solution is contained in the implementation section
} 
determined by $w_{12}, w_{15}$, and $w_{16}$, i.e. once you know the number of codewords of weight 12,15 , and 16, you can compute the number of codewords of weight 19 . This has the effect of imposing additional conditions on the incidence matrix of a finite projective plane. Specifically, a particular codeword of weight $i$ is equivalent to a configuration $\sigma$ of $i$ points in the plane. Applying these results allows for the lines of the plane to be partitioned into $C^{i}{ }_{k}$ each containing the lines incident with $\boldsymbol{\sigma}$ in $\boldsymbol{k}$ points. Therefore, if the number of code words of a given weight $\boldsymbol{i}$ is greater than 0 , the lines of the plane will have to satisfy the incidence with the configuration $\sigma$ of $i$ points as specified by the $C^{i}{ }_{k}$. This has the effect of reducing both the possible starting configurations of an incidence matrix and the number of possible branches at each partial solution point in the search tree.

With the following strategy, it becomes possible to settle the existence of a projective plane of order $10[3,4,5,6,7,8]$ :

- determine $w_{12}, w_{15}$, and $w_{16}$ so that $C$ 's weight enumerator is solved (it is possible that a plane may be built in this determination, in which case we are done);

- use the weight enumerator to compute $\boldsymbol{w}_{19}$, i.e. the number of codewords of weight 19 in $C$;

- attempt to build the incidence matrix complying to the restrictions imposed by the $\mathrm{C}^{19}{ }_{k}$, i.e. the determined incidence of the lines with the configuration $\sigma$ of 19 points in the plane; 
- if the incidence matrix is successfully built, then a projective plane of order 10 exists;

- or else we arrive at a contradiction regarding the $w_{19}$ computed from the weight enumerator, and a projective plane of order 10 does not exist.

We now proceed with a detailed analysis of the $w_{12}, w_{15}, w_{16}$, and $w_{19}$ configurations [8]. 


\subsection{5-point configurations}

Assume there exists a codeword of weight 15 in $\boldsymbol{C}$. We can number the corresponding points in the associated plane 1 to 15 , and denote them as set $M_{15}$. Based on lemma 1.3.3, we know that any line $\ell$ of the plane will intersect $M_{15}$ in an odd number of points, specifically 1,3 or 5 points.

Proof. $\left|\boldsymbol{M}_{\mathbf{1 5}}+\ell\right|=15+11-2\left|\boldsymbol{M}_{\mathbf{1 5}} \cap \ell\right| \geq 12 \rightarrow\left|\boldsymbol{M}_{\mathbf{1 5}} \cap \ell\right| \leq 7$.

If $\left|M_{15} \cap \ell\right|=7$, then $\boldsymbol{M}_{15}+\ell$ is a hyperoval $\mathrm{o}$. But then $|\ell \cap 0|=4$, contradicting the definition of a hyperoval (i.e. intersection in 0 or 2 points as per lemma 1.3.6).

Next, let $b_{1}, b_{3}$ and $b_{5}$ denote the number of lines (light, medium and heavy lines respectively) that intersect $\boldsymbol{M}_{15}$ in 1,3 or 5 points respectively. We can determine $b_{1}=90, b_{3}=15$ and $b_{5}=6$ by solving the following system of equations:

$$
\begin{gathered}
b_{1}+b_{3}+b_{5}=111 \quad \text { (a count of the lines of the planes) } \\
\left(\begin{array}{l}
3 \\
2
\end{array}\right) b_{3}+\left(\begin{array}{c}
5 \\
2
\end{array}\right) b_{5}=\left(\begin{array}{c}
15 \\
2
\end{array}\right) \quad\left(\text { a count of the pars of points of } M_{15}\right) \\
\left.10 b_{1}+8 b_{3}+6 b_{5}=(111-15) 11 \quad \text { (a count of }(\ell, p), p \in \ell, p \notin M_{15}\right)
\end{gathered}
$$


Hence the incidence matrix's starting configuration equivalent to a codeword of weight 15 is a $6 \times 15$ submatrix, corresponding to 6 lines intersecting the $\boldsymbol{M}_{\mathbf{1 5}}$ in 5 points and the 15 points of the configuration. 


\subsection{2-point configurations}

Assume there exists a codeword of weight 12 in $C$. We can number the corresponding points in the associated plane 1 to 12 , and denote them as set $M_{12}$. Based on a lemma 1.3.3, we know that any line $\ell$ of the plane will intersect $M_{12}$ in an even number of points. Furthermore, it was shown that codewords of weight 12 are equivalent to hyperovals of the order 10 plane, and that these intersect a line in 0 or 2 points. By definition of a projective plane, pairs of points occur uniquely on a line. In set $\boldsymbol{M}_{12}$ we have 66 pairs which correspond to the lines which intersect the codeword in 2 points, hence 66 lines intersect $\boldsymbol{M}_{\mathbf{1 2}}$ in 2 points with the remaining 45 lines of the plane intersecting in 0 points. Hence the incidence matrix's starting configuration equivalent to a codeword of weight 12 is a $66 \times 12$ submatrix. This structure can be extended to a full 21 lines, uniquely up to isomorphism. However, extending it further beyond 30 lines becomes as computationally intensive as the methods presented in Section 1.1. 


\subsection{6-point configurations}

Assume there exists a codeword of weight 16 in $\mathbf{C}$. We can number the corresponding points in the associated plane 1 to 16 , and denote them as set M16. Based on a lemma 1.3.3, we know that any line $\ell$ of the plane will intersect $\boldsymbol{M}_{\mathbf{1 6}}$ in an even number of points. $\left|\boldsymbol{M}_{\mathbf{1 6}}+\ell\right|=16-\boldsymbol{s}+(11-\boldsymbol{s})$, where $\boldsymbol{s}$ equals the number of points that are common to $\boldsymbol{M}_{16}$ and $\ell$. For $\boldsymbol{s}=6$ or $10,\left|\boldsymbol{M}_{16}+\ell\right|=$ 15 or 7 respectively, meaning that the intersection is a vector of weight 15 or 7 . We have already shown that there do not exist any codewords of weight less than 11(other than the all-zero vector). In 1973, MacWilliams, Sloane and Thompson proved that $\boldsymbol{M}_{15}=0$ (their proof will be presented in section 2.2 , and verified in section 3.7). It remains to consider $s=8$, for which $\left|M_{16}+\ell\right|=11$, then the intersection is an actual line of the plane. But two lines can only have one point in common which would yield an $\boldsymbol{M}_{\mathbf{2 0}}$, unless the two lines are identical then we have the all-zero vector. Hence, any line $\ell$ of the plane will intersect $\boldsymbol{M}_{\mathbf{1 6}}$ in 0,2 or 4 points.

Next let $b_{0}, b_{2}$ and $b_{4}$ denote the number of lines that intersect $\boldsymbol{M}_{\mathbf{1 6}}$ in 0,2 or 4 points respectively. We can determine $b_{0}=31, b_{2}=72$ and $b_{4}=8$ by solving the following system of equations: 


$$
\begin{gathered}
b_{0}+b_{2}+b_{4}=111 \\
2 b_{2}+4 b_{4}=176 \quad\left(\text { a count of }(\ell, p), p \in \ell, p \in M_{16}\right) \\
\left(\begin{array}{c}
2 \\
2
\end{array}\right) b_{2}+\left(\begin{array}{c}
4 \\
2
\end{array}\right) b_{4}=\left(\begin{array}{c}
16 \\
2
\end{array}\right)
\end{gathered}
$$

Hence the incidence matrix's starting configuration equivalent to a codeword of weight 16 is a $8 \times 16$ submatrix. 


\subsection{9-point configurations}

Assume there exists a codeword of weight 19 in $\boldsymbol{C}$. We can number the corresponding points in the associated plane 1 to 19 , and denote them as set M19. Based on lemma 1.3.3, we know that any line $\ell$ of the plane will intersect $M_{19}$ in an odd number of points. Suppose that $M_{19}$ and $\ell$ have 11 points in common then $\left|\boldsymbol{M}_{19}+\ell\right|=8$, but we have already shown that there do not exist any codewords of weight less than 11 (other than the all-zero vector).

Next, suppose that $\boldsymbol{M}_{19}$ and $\ell$ have at most 9 points in common then $\left|\boldsymbol{M}_{19}+\ell\right|=$ 12 , and the resultant vector is a hyperoval and hence we name it a hyperovaltype $\boldsymbol{M}_{\mathbf{1 9}}$. For any given hyperoval we have 66 distinct Hyperoval-type $\mathbf{M}_{\mathbf{1 9}}$, since we may add to the hyperoval any one of its 66 secants and this produces an $M_{19}$ with a unique line intersecting in 9 points.

Next, suppose that $\boldsymbol{M}_{19}$ and $\ell$ have at most 7 points in common, then $\left|\boldsymbol{M}_{19}+\ell\right|=$ 16 and the resultant vector is an $\boldsymbol{M}_{\mathbf{1 6}}$ (hence we name it a 16-type $\boldsymbol{M}_{\mathbf{1 9}}$ ). Let $b_{1}$, $b_{3}, b_{5}$ and $b_{7}$ denote the number of lines that intersect a 16-type $\boldsymbol{M}_{19}$ in $1,3,5$ or 7 points respectively. Note that $b_{7}$ must equal 1 : 
- $b_{7} \geq 3$ produces a negative value (a negative number of lines) for $b_{5}$ in the system of equations below;

- Although $b_{7}=2$ produces consistent positive values $\left(b_{1}=66, b_{3}=43, b_{5}=0\right.$ and $b_{7}=2$ ) in the equations below, we have computed that it is impossible to find the 43 lines intersecting the $M_{19}$ in three points without violating the definition of a finite plane (some pair of points will occur on more than one line).

We can determine $b_{1}=67, b_{3}=40, b_{5}=3$ and $b_{7}=1$ by solving the following system of equations:

$$
\begin{array}{r}
b_{1}+b_{3}+b_{5}+b_{7}=111 \\
b_{1}+3 b_{3}+5 b_{5}+7 b_{7}=209 \\
\left(\begin{array}{l}
3 \\
2
\end{array}\right) b_{3}+\left(\begin{array}{c}
5 \\
2
\end{array}\right) b_{5}+\left(\begin{array}{c}
7 \\
2
\end{array}\right) b_{7}=171
\end{array}
$$

Hence the incidence matrix's starting configuration equivalent to a 16 -type $\boldsymbol{M}_{\mathbf{1 9}}$ is a $4 \times 19$ submatrix.

To obtain a 16 -type $\boldsymbol{M}_{19}$ from an $\boldsymbol{M}_{\mathbf{1 6}}$, we simply add one of the eight lines that intersects the $M_{16}$ in four points. The aforementioned line is the unique line of the $M_{19}$ which contains 7 points. Hence each $M_{16}$ yields 8 16-type $M_{19}$ s. 
Finally we must consider the possibility of an $M_{19}$ which intersects all lines $\ell$ in less than 7 points (i.e. a primitive $M_{19}$ ). Let $b_{1}, b_{3}$ and $b_{5}$ denote the number of lines that intersect a primitive $\boldsymbol{M}_{19}$ in 1,3 , or 5 points respectively. We can determine $b_{1}=68, b_{3}=37$, and $b_{5}=6$ by solving the following system of equations:

$$
\begin{array}{r}
b_{1}+b_{3}+b_{5}=111 \\
b_{1}+3 b_{3}+5 b_{5}=209 \\
\left(\begin{array}{l}
3 \\
2
\end{array}\right) b_{3}+\left(\begin{array}{l}
5 \\
2
\end{array}\right) b_{5}=\left(\begin{array}{c}
19 \\
2
\end{array}\right)
\end{array}
$$

Hence the incidence matrix's starting configuration equivalent to a primitive $\boldsymbol{M}_{\mathbf{1 9}}$ is a $6 \times 19$ submatrix. 
We can now refine the relation obtained from the MacWilliams identity to compute just the primitive $\boldsymbol{M}_{\mathbf{1 9}}$ 's (denoted $\boldsymbol{P}_{19}$ ) [8]:

$$
w_{19}=24675+141 w_{12}-27 w_{15}+7 w_{16}
$$

The weight-19 codewords consist of:

$$
W_{19}=\left|P_{19}\right|+66^{*}\left|M_{12}\right|+8^{*}\left|M_{16}\right|
$$

Hence we have:

$$
\left|\boldsymbol{P}_{19}\right|+66^{*}\left|\boldsymbol{M}_{\mathbf{1 2}}\right|+8^{*}\left|\boldsymbol{M}_{16}\right|=24675+141 \boldsymbol{w}_{\mathbf{1 2}}-27 \boldsymbol{w}_{15}+7 \boldsymbol{w}_{16}
$$

Therefore we obtain the following expression of primitive weight-19 codewords:

$$
\left|P_{19}\right|=24675+75^{*}\left|M_{12}\right|-27^{*}\left|M_{15}\right|-\left|M_{16}\right|
$$

Note that $w_{12}=\left|M_{12}\right|, w_{15}=\left|M_{15}\right|$ and $w_{16}=\left|M_{16}\right|$ (since for each there is only one possible configuration). 


\section{Chronology of the Solution}

This section details the timeline of all research which determined the nonexistence of the projective plane of order 10. For the most part, the theory necessary has already been provided in the previous section and substantiation of empirical results (i.e. confirmation) will be presented in the $3^{\text {rd }}$ section.

\subsection{Assmus and Matson}

In the late 1960's, Edward F. Assmus Jr. and Harold F. Mattson Jr. were employed by GTE Sylvania and under contract with the United States Air Force to work on the algebraic theory of codes. It was during this time that it occurred to Assmus to analyze the code associated to the incidence matrix of the hypothetical projective plane of order 10 .

Assmus presented this idea at the conference "Combinatorial Aspects of Finite Geometries" at Oberwolfach, Germany held from March 30 to April 4 1970. The presentation sparked a lot of interest in the combinatorial community, many thinking that it could lead to a solution to the question of existence of the plane of order 10. 
On the $15^{\text {th }}$ of October 1970, Assmus and Matson published a paper entitled "Algebraic Theory of Codes II" for the Air Force Cambridge Research Laboratories (which remained classified for two decades). In it is a section entitled "On the possibility of a projective plane of order ten". Here they provided a detailed analysis of the code associated to a plane of order 10 . Also contained is an alternate proof, using coding theory, of the non-existence of a projective plane of order 6 .

While Assmus and Mattson's work does not settle the existence question, it lays down the foundation for all the work that is to follow.

\subsection{MacWilliams, Sloane and Thompson}

In 1973, MacWilliams, Sloane and Thompson published a paper which determined the number of codewords of weight 15 in $C$, the plane of order 10's error correcting code [5]. It also contained a concrete analysis of $\boldsymbol{C}$, emphasizing the aspects which allow the development of efficient techniques and computer programs to determine the number of codewords of a given weight. A summary of the work follows. 
We begin by constructing a $6 \times 15$ submatrix $A 1$, which represents the partial plane satisfying $b_{5}=6$ of the configuration parameters presented in the previous section. Each pair of (heavy) lines of $A 1$ will intersect in a point $P$ in the $M_{15}$. For suppose that there exists an intersection point $P$ not in the $\boldsymbol{M}_{15}$ : then we will use 10 points of the $M_{15}$. The other 9 lines through $P$ must each contain at least one more point of the $\boldsymbol{M}_{15}$, but there would only be 5 points of the $\boldsymbol{M}_{15}$ left.

Furthermore any three or more lines of $A 1$ cannot have a common point. For suppose that there exist three lines of $A 1$ that all intersect in a point $P$ in the $M_{15}$. This will use up 13 points of the $M_{15}$. We will then require one point each from the first three lines and the two remaining unused points to construct the $4^{\text {th }}$ line. But then we cannot construct a $5^{\text {th }}$ line, since we can only use 4 points ance from the first 4 lines and we have no more unused points remaining. This gets even worse for more than 3 lines.

Hence, each of the 15 points of the $M_{15}$ uniquely determines an intersection between 2 lines of submatrix $A 1$. Without loss of generality, the first six lines can be set in the following manner:

$\begin{array}{ccccc}1 & 2 & 3 & 4 & 5 \\ 1 & 6 & 7 & 8 & 9 \\ 2 & 6 & 10 & 11 & 12 \\ 3 & 7 & 10 & 13 & 14 \\ 4 & 8 & 11 & 13 & 15 \\ 5 & 9 & 12 & 14 & 15\end{array}$


Next, we address the lines which intersect the $\boldsymbol{M}_{\mathbf{1 5}}$ in three points. In Section 1.4 we determined that $b_{3}=15$. Up to isomorphism, MacWilliams et al. found only one way to complete these (medium) lines:

$\begin{array}{ccc}1 & 10 & 15 \\ 1 & 11 & 14 \\ 1 & 12 & 13 \\ 2 & 7 & 15 \\ 2 & 8 & 14 \\ 2 & 9 & 13 \\ 3 & 6 & 15 \\ 3 & 8 & 12 \\ 3 & 9 & 11 \\ 4 & 6 & 14 \\ 4 & 7 & 12 \\ 4 & 9 & 10 \\ 5 & 6 & 13 \\ 5 & 7 & 11 \\ 5 & 8 & 10\end{array}$

We label these 15 partial lines, together with the first six an $A 2$. Next, since each of the first six lines intersects each other already, we can add 6 more points to each line shown below:

\begin{tabular}{ccccc|cccccc}
1 & 2 & 3 & 4 & 5 & 76 & 77 & 78 & 79 & 80 & 81 \\
1 & 6 & 7 & 8 & 9 & 82 & 83 & 84 & 85 & 86 & 87 \\
2 & 6 & 10 & 11 & 12 & 88 & 89 & 90 & 91 & 92 & 93 \\
3 & 7 & 10 & 13 & 14 & 94 & 95 & 96 & 97 & 98 & 99 \\
4 & 8 & 11 & 13 & 15 & 100 & 101 & 102 & 103 & 104 & 105 \\
5 & 9 & 12 & 14 & 15 & 106 & 107 & 108 & 109 & 110 & 111 \\
\hline
\end{tabular}

Finally, there remain 60 unused points. Each of these must meet exactly 2 of the medium lines $\left(15^{\star} 8 / 60=2\right)$. For suppose 3 lines pass through one of these points, say $P$. This will use up 9 points of the $\boldsymbol{M}_{15}$. Then $P$ can only occur on $15-9=6$ 
more lines in the plane, i.e. $P$ will only meet 9 lines. Up to isomorphism, MacWilliams et al. found only one way to complete the first 21 lines:

\begin{tabular}{ccccccccccc}
1 & 2 & 3 & 4 & 5 & 76 & 77 & 78 & 79 & 80 & 81 \\
1 & 6 & 7 & 8 & 9 & 82 & 83 & 84 & 85 & 86 & 87 \\
2 & 6 & 10 & 11 & 12 & 88 & 89 & 90 & 91 & 92 & 93 \\
3 & 7 & 10 & 13 & 14 & 94 & 95 & 96 & 97 & 98 & 99 \\
4 & 8 & 11 & 13 & 15 & 100 & 101 & 102 & 103 & 104 & 105 \\
5 & 9 & 12 & 14 & 15 & 106 & 107 & 108 & 109 & 110 & 111 \\
\hline 1 & 10 & 15 & 46 & 47 & 52 & 53 & 58 & 59 & 64 & 65 \\
1 & 11 & 14 & 40 & 41 & 55 & 56 & 61 & 62 & 70 & 71 \\
1 & 12 & 13 & 43 & 44 & 49 & 50 & 67 & 68 & 73 & 74 \\
2 & 7 & 15 & 32 & 33 & 34 & 36 & 61 & 63 & 67 & 69 \\
2 & 8 & 14 & 25 & 26 & 38 & 39 & 58 & 60 & 73 & 75 \\
2 & 9 & 13 & 22 & 23 & 28 & 29 & 64 & 66 & 70 & 72 \\
3 & 6 & 15 & 29 & 30 & 37 & 38 & 49 & 51 & 56 & 57 \\
3 & 8 & 12 & 16 & 17 & 35 & 36 & 46 & 48 & 71 & 72 \\
3 & 9 & 11 & 18 & 20 & 31 & 33 & 52 & 54 & 74 & 75 \\
4 & 6 & 14 & 22 & 24 & 34 & 35 & 43 & 45 & 53 & 54 \\
4 & 7 & 12 & 18 & 21 & 37 & 39 & 40 & 42 & 65 & 66 \\
4 & 9 & 10 & 16 & 19 & 26 & 27 & 55 & 57 & 68 & 69 \\
5 & 6 & 13 & 25 & 27 & 31 & 32 & 41 & 42 & 47 & 48 \\
5 & 7 & 11 & 17 & 19 & 28 & 30 & 44 & 45 & 59 & 60 \\
5 & 8 & 10 & 20 & 21 & 23 & 24 & 50 & 51 & 62 & 63
\end{tabular}

The remaining 90 (light) lines of the plane are easy to determine. Each of the points of the $\boldsymbol{M}_{\mathbf{1 5}}$ has occurred on 5 lines and intersected every other point of the $M_{15}$, and hence must occur independently on 6 more lines (and $6^{\star} 15=90$ ).

At this point, MacWilliams et al. turned to a computer in an attempt to extend the above incidence matrix to a full plane of order 10. They began with the 6 light lines containing point 1 of the $\boldsymbol{M}_{15}$. We must add 10 points to each line, i.e. $66^{\star} 10=60$ distinct points. These points must come from: 
- One point from the last 6 of each of the $3^{\text {rd }}$ to $6^{\text {th }}$ lines (e.g. $88,94,100$ and 106 is a valid combination);

- 6 points among those from lines 10 to 21 which have not already occurred on lines 7 to 9 , and which have not yet occurred pairwise on any other line (e.g 17, 18, 24, 26, 29 and 32 is a valid combination).

Up to isomorphism, MacWilliams et al. found 1021 extensions of the incidence matrix to the 6 light lines containing point 1 of the $\boldsymbol{M}_{\mathbf{1 5}}$. It should be noted that they did not perform isomorph rejection computationally, but rather determined that the $M_{15}$ is invariant under a group $G$ isomorphic to the symmetric group $\zeta_{6}$, and then computed G's group actions (i.e. collineation on the heavy and medium lines). This allowed the program to choose representatives from the orbits of $G$.

The next step is to extend another set of light lines. Those with point 10 of the $M_{15}$ were chosen because of greater constraints with the light lines containing point 1 , resulting in a smaller backtrack search. The maximum number of surviving extensions to the light lines containing point 10 was always less than 100. It was found that the incidence matrix could not be extended beyond 2 more sets of light lines (in points 11 and 15). This required about 3 hours of computer time on a General Electric 635. 
Since an incidence matrix allowing for a 15-point configuration could not be extended to a full plane, the conclusion is that the code associated to a projective plane of order 10 does not contain any codewords of weight 15 , i.e.

$$
\left|M_{15}\right|=0 \rightarrow\left|P_{19}\right|=24675+75^{\star}\left|M_{12}\right|-\left|M_{16}\right|
$$

Note that if we hadn't determined that $\left|M_{15}\right|=0$, we would also not be able to conclude that there exist only primitive $\boldsymbol{M}_{16}$ s (see Section 1.6 on 16 -point configurations for details). 


\subsection{Carter}

In his $1974 \mathrm{PhD}$ thesis, Carter tackled the problem of determining the number of codewords of weight 16 in $C$, the plane of order 10's error correcting code [6]. As we will see below, solving the weight-16 configurations is rather more complicated than weight 15 . Hence Carter devotes a lot of effort in developing efficient algorithmic techniques for backtrack searches in general, and develops programs that are very specific to solving the weight- 16 case in his thesis. Also, it is where the first detailed analysis of the weight-16 configurations (seen in the section 1.6) appeared in the literature.

As we have seen an $\boldsymbol{M}_{16}$ starting configuration, $A 1$, consists of 16 points on 8 (heavy) lines. Each of the heavy lines contains four points of the $\mathbf{M}_{\mathbf{1 6}}$, with each point occurring on 2 heavy lines. Carter determined that, up to isomorphism, there are six different possibilities for an $A 1$ matrix: 


$\begin{array}{cccccccccccc}1 & 2 & 3 & 4 & 1 & 2 & 3 & 4 & 1 & 2 & 3 & 4 \\ 1 & 5 & 6 & 7 & 1 & 5 & 6 & 7 & 1 & 5 & 6 & 7 \\ 2 & 8 & 9 & 10 & 2 & 5 & 8 & 9 & 2 & 5 & 8 & 9 \\ 3 & 11 & 12 & 13 & 3 & 6 & 8 & 10 & 3 & 6 & 10 & 11 \\ 4 & 14 & 15 & 16 & 4 & 11 & 12 & 13 & 4 & 12 & 13 & 14 \\ 5 & 8 & 11 & 14 & 7 & 11 & 14 & 15 & 7 & 12 & 15 & 16 \\ 6 & 9 & 12 & 15 & 9 & 12 & 14 & 16 & 8 & 10 & 13 & 15 \\ 7 & 10 & 13 & 16 & 10 & 13 & 15 & 16 & 9 & 11 & 14 & 16 \\ & & & & & & & & & & & \\ 1 & 2 & 3 & 4 & 1 & 2 & 3 & 4 & 1 & 2 & 3 & 4 \\ 1 & 5 & 6 & 7 & 1 & 5 & 6 & 7 & 1 & 5 & 6 & 7 \\ 2 & 5 & 8 & 9 & 2 & 5 & 8 & 9 & 2 & 5 & 8 & 9 \\ 3 & 6 & 10 & 11 & 3 & 6 & 10 & 11 & 3 & 6 & 10 & 11 \\ 4 & 12 & 13 & 14 & 8 & 12 & 13 & 14 & 8 & 12 & 13 & 14 \\ 8 & 12 & 15 & 16 & 10 & 12 & 15 & 16 & 10 & 12 & 15 & 16 \\ 7 & 10 & 13 & 15 & 4 & 7 & 13 & 15 & 4 & 9 & 13 & 15 \\ 9 & 11 & 14 & 16 & 9 & 11 & 14 & 16 & 7 & 11 & 14 & 16\end{array}$

Recall that in the weight-15 case, there was only one possibility for its $A 1$.

Next we must address the 72 (medium) lines that intersect the $\boldsymbol{M}_{\mathbf{1 6}}$ in 2 points, an $A 2$ which is unique to its starting configuration, i.e. it is merely an exercise in computing the remaining 72 pairs of the $\boldsymbol{M}_{\mathbf{1 6}}$ which are not covered by the heavy lines. Carter ignores the 31 (light) lines in his programs, i.e. those that do not intersect the $\boldsymbol{M}_{16}$. So it remains a question of attempting to extend each of the six $80 \times 16$ sub-matrices to a full incidence matrix.

In terms of complexity relative to the weight-15 case (which had only one possibility for the first full 21 lines) if one attempts to extend the weight-16 A2 lineby-line, the non-isomorphic instances explode to essentially intractable proportions shortly after the $21^{\text {st }}$ line. Hence Carter extends the incidence matrix 
by adding complete columns ${ }^{\Psi}$ (up to the $80^{\text {th }}$ row), since this technique restricts the growth of the backtrack search more effectively.

Nevertheless, Carter can only complete the search for 4 of the 6 starting configurations (and part of a fifth) given the computational "horsepower" at the time. None of these cases extend to a full plane after about 100 hours of computer time on a CDC 7600 (in contrast to the three hours required to solve the entire weight-15 case).

\footnotetext{
${ }^{\Psi}$ A more thorough presentation of techniques necessary to solve the weight 16 case will be presented in section 2.5 .
} 


\subsection{Thompson}

So far, in analyzing the weight-15 and 16 cases, we have seen that they both reduce to a low number of small starting configurations which can, for the most part, be handled by a computer. But as we saw for the analysis of 12-point configurations, the incidence matrix of the starting configuration consists of 66 lines which each intersect the $M_{12}$ in 2 points. Attempting to extend the incidence matrix from this point is not much less time-consuming than a straightout brute force backtrack search of the entire incidence matrix (which we have seen to be too time-consuming).

Thompson posed the following question: "Does there exist a set $S$ of 99 fixed point free involutions on 12 points such that for each involution (ab).(cd) which moves just 4 points, there is a unique $s \in S$ which has $\{a, b\}$ and $\{c, d\}$ as orbits?" [23]. This question is important because if it is answered in the negative, then hyperovals do not exist in a projective plane of order 10 . Thompson performs a thorough group theory analysis and initiates a search, but leaves it to others to solve. However, he is the first to propose an effective strategy to determine if hyperovals (12-point configurations) exist in a plane of order 10. 


\subsection{Lam, Thiel and Swiercz}

Between 1981 and 1989, Lam, Thiel and Swiercz performed all the remaining steps necessary to solve the existence problem. That is, they determined that there exist no hyperovals, nor (primitive) $\boldsymbol{M}_{\mathbf{1 6}}$ 's, nor $\boldsymbol{P}_{19}$ 's in a plane of order 10 , effectively proving the non-existence of a projective plane of order $10 \otimes$.

Lam et al. began by reformulating Thompson's analysis into a form that can be efficiently handled by a computer [28]. Recall that a hyperoval o consists of 12 points $(1,2 \ldots ., 12)$, and every line of the plane contains either 2 points (66 lines) or zero points (45 lines) of $o$. Furthermore, each of the 99 points not on $o$ is on exactly 6 of the 66 lines. These lines and points can be labeled by special involutions in the symmetric group $S$ on 12 symbols. Let $C_{2}$ and $C_{6}$ represent the set of permutations with cycle type $2^{2} 1^{8}$ and $2^{6}$, and define the term "covers" to mean the following:

( $\left.\alpha \in C_{6}\right)$ covers $\left(\beta \in C_{2}\right)$ if the two 2-cycles of $\beta$ appear among the six 2-

cyles of $\alpha$, e.g. 214365871091211 covers 214356789101112

A hyperoval is then equivalent to a subset $D \subset C_{6}$ satisfying the following:

1. $|D|=99$;

2. $\forall \beta \in C_{2}, \exists$ unique $\alpha \in D$ such that $\alpha$ covers $\beta$. 
It then becomes a question of constructing a backtrack search to find $99 \alpha s$ which satisfy the two properties above. In order to reduce the time required to execute the search, Lam et al. considered only the non-isomorphic permutations of $C_{6}$. They also determined a search order on elements of $C_{6}$ which maximized constraints. In 183 days of computing time, they found that $|D|$ could never exceed 51 , hence hyperovals cannot exist in a plane of order 10 , and therefore:

$$
\left|M_{12}\right|=0 \rightarrow\left|P_{19}\right|=24675-\left|M_{16}\right|
$$

Lam et al. then undertook the task of fully solving $\boldsymbol{M}_{\mathbf{1 6}}[26]$. As discussed earlier, the extension of the incidence matrix for an $\boldsymbol{M}_{16}$ starting configuration is accomplished by adding full columns (up to the $80^{\text {th }}$ line) rather than adding full lines for reasons of computational efficiency. We now detail this extension process in more detail, starting from the following $\boldsymbol{M}_{\mathbf{1 6}}$ starting configuration:

$\begin{array}{cccc}1 & 2 & 3 & 4 \\ 1 & 5 & 6 & 7 \\ 2 & 5 & 8 & 9 \\ 3 & 6 & 10 & 11 \\ 4 & 12 & 13 & 14 \\ 8 & 12 & 15 & 16 \\ 7 & 10 & 13 & 15 \\ 9 & 11 & 14 & 16\end{array}$


In the first step we add the balance of required points to line 1 (denoted block 1), as follows (note that the last 3 lines must each include a point from block 1 in order to be incident with line 1):

$\begin{array}{ccccccccccc}1 & 2 & 3 & 4 & 17 & 18 & 19 & 20 & 21 & 22 & 23 \\ 1 & 5 & 6 & 7 & & & & & & & \\ 2 & 5 & 8 & 9 & & & & & & & \\ 3 & 6 & 10 & 11 & & & & & & & \\ 4 & 12 & 13 & 14 & & & & & & \\ 8 & 12 & 15 & 16 & 17 & & & & & \\ 7 & 10 & 13 & 15 & 18 & & & & & & \\ 9 & 11 & 14 & 16 & 19 & & & & & & \end{array}$

Next we address the 72 medium lines (with 2 points of the $M_{16}$ ) relative to the block-1 points we just added: any line that does not contain points 1 through 4 will have a point from block 1 added to it, subject to the constraints of a projective plane. We then add the balance of required points to line 2 (denoted block 2), as follows:

$\begin{array}{ccccccccccc}1 & 2 & 3 & 4 & 17 & 18 & 19 & 20 & 21 & 22 & 23 \\ 1 & 5 & 6 & 7 & 24 & 25 & 26 & 27 & 28 & 29 & 30 \\ 2 & 5 & 8 & 9 & & & & & & & \\ 3 & 6 & 10 & 11 & & & & & & & \\ 4 & 12 & 13 & 14 & 24 & & & & & & \\ 8 & 12 & 15 & 16 & 17 & 25 & & & & & \\ 7 & 10 & 13 & 15 & 18 & & & & & & \\ 9 & 11 & 14 & 16 & 19 & 26 & & & & & \end{array}$

Once again we address the 72 lines relative to the block- 2 points in the same manner as above. And we continue to add blocks until the 8 blocks are all complete (along with the medium lines). Now with none of the cases that Carter 
handled in his thesis was it possible to extend beyond block 5 . However it is not possible to address the additional cases not solved by Carter in this algorithmic manner in a reasonable amount of computer time.

The additional cases are the ones that Lam et al. solved by splitting into five subcases which were each addressed by highly specialized programs that targeted the individual search properties of each of these subcases. We will present here the method used to solve one of those subcases.

Consider the two-distinguished points (as denoted by Lam et al.) subcase:

\begin{tabular}{|c|c|c|c|c|c|c|c|c|c|c|c|}
\hline 1 & 2 & 3 & 4 & 17 & 19 & 20 & 21 & 22 & 23 & 24 & Block 1 \\
\hline 5 & 6 & 7 & 8 & 17 & & & & & & & \\
\hline 9 & 10 & 11 & 12 & 17 & & & & & & & \\
\hline 13 & 14 & 15 & 16 & 17 & 37 & 38 & 39 & 40 & 41 & 42 & Block 4 \\
\hline 1 & 5 & 9 & 13 & 18 & 43 & 44 & 45 & 46 & 47 & 48 & Block 5 \\
\hline 2 & 6 & 10 & 14 & 18 & 49 & 50 & 51 & 52 & 53 & 54 & Block 6 \\
\hline 3 & 7 & 11 & 15 & 18 & 25 & 26 & 27 & 28 & 29 & 30 & Block 2 \\
\hline 4 & 8 & 12 & 16 & 18 & 31 & 32 & 33 & 34 & 35 & 36 & Block 3 \\
\hline 5 & 10 & 19 & 25 & 31 & 37 & 55 & 56 & 57 & 58 & 59 & Special \\
\hline
\end{tabular}

The $M_{16}$ starting configuration is enclosed in the box. The first four lines are not incident with each other, nor are the last four (incident with each other). We add two (distinguished) points which address the incidence in one step (note that the other two possible incidence patterns are handled in other subcases). We then add points to the blocks as before, however these now contain only 6 points 
instead of 7. Also Lam et al. determined with extensive estimation runs the optimal order of lines (hence the column indicating the block order). In addition, there is a special line (the medium line which contains points 5 and 10): points 19 , 25, 31 and 37 address its outside (the $\boldsymbol{M}_{16}$ ) incidence with heavy lines, and the others are its additional required points. Finally, we address the incidence of the 71 medium lines with points 19 to 59 above relative to the following order:

- block 1;

- The eight remaining points on the special line;

- blocks 2 through 6 .

In addition to the algorithmic techniques above, Lam et al. make extensive use of symmetry exhibited by the blocks. This greatly reduces the number of isomorphic instances that would otherwise needlessly be computed by the program. It took about 80 days of computer time to determine that the two-distinguished points and the other subcases cannot be extended to a plane of order 10 . Therefore Lam et al. were able to conclude that:

$$
\left|M_{16}\right|=0 \rightarrow\left|P_{19}\right|=24675
$$

In other words, the weight enumerator is now solved, i.e. a plane of order 10 must contain $24,675 \boldsymbol{P}_{19}$ 's. Hence beginning with at least one of the $\boldsymbol{P}_{19}$ starting configurations for the incidence matrix, we must be able to extend it to a full plane 
or else the assumption of the existence of a projective plane of order 10 has led to a flawed weight enumerator and must be false.

Although a lot of computer time was spent on determining $M_{15}, M_{12}$ and $M_{16}$, Lam et al. completed the most time-consuming step to date in order to solve $\boldsymbol{P}_{19}$ $[3,27,30]$. The number of $\boldsymbol{P}_{19}$ starting configurations grows significantly to 66 , but 21 of these cases are dismissed due to theoretical reasons (most of them because the incidence matrix can not be extended to the medium lines). While the $M_{15}$ and $M_{16}$ cases had a one-to-one relationship when extending the initial matrix to include the medium lines (i.e. each heavy line starting configuration $A 1$ resulted in just one instance of the medium lines $A 2$ ), the $45 \boldsymbol{P}_{19}$ starting configurations result in a total of over 600,000 non-isomorphic A2s. For each of these $A 2 s$ there is a single instance of the light lines extension, i.e. A3. And from this point, it is "simply" a matter of extending the incidence matrix by using the same block method (adding entire columns) as with the $\mathbf{M}_{\mathbf{1 6}}$ 's.

Lam et al. distinguished between two cases of $\boldsymbol{P}_{19}$ starting configurations. The first case has some intersections outside of the points of the $\boldsymbol{P}_{19}$. Here is one example: 


$\begin{array}{ccccccccccc}1 & 2 & 3 & 4 & 5 & 20 & 21 & 22 & 23 & 24 & 25 \\ 1 & 6 & 7 & 8 & 9 & 26 & 27 & 28 & 29 & 30 & 31 \\ 1 & 10 & 11 & 12 & 13 & 32 & 33 & 34 & 35 & 36 & 37 \\ 2 & 6 & 14 & 15 & 16 & & & & & & \\ 3 & 7 & 14 & 17 & 18 & & & & & & \\ 4 & 8 & 15 & 17 & 19 & 32 & 38 & 39 & 40 & 41 & 42\end{array}$

Note that the $3^{\text {rd }}$ and $6^{\text {th }}$ line do not intersect in a point of the $\boldsymbol{P}_{19}$. This means that their blocks must share a point outside of the $\boldsymbol{P}_{19}$. Hence when determining the incidence of the medium and light lines with these blocks, only $11(6+5)$ points are considered rather than 12 (for say lines 1 and 2). When a starting configuration has outside intersections (the more the better) the size of the computation tends to be smaller. Lam called these the "easy" cases. Of the 45 viable starting configurations, 37 have outside intersections. These were solved in 800 days of computing time. None of them could be extended beyond block 4 .

There remained the eight "difficult" cases, the ones without outside intersections. Here is one example:

$\begin{array}{ccccc}1 & 2 & 3 & 4 & 5 \\ 1 & 6 & 7 & 8 & 9 \\ 2 & 6 & 10 & 11 & 12 \\ 3 & 7 & 10 & 13 & 14 \\ 4 & 8 & 11 & 13 & 15 \\ 5 & 9 & 12 & 14 & 15\end{array}$

It was estimated that using the "standard" block technique to solve these cases would take about 10 years of computing time. At this point Lam decided to request the use of a Cray super-computer. This however meant that new 
programs had to be developed to take advantage of the special capabilities of the Cray [30]. In a nutshell, the difference between a standard computer (like a Vax on which most of Lam's computations were performed) and the Cray was that you could perform the same operation on multiple "vectors" at once. This did not apply very well to a traditional backtrack program where only one choice is considered at a time.

The basic concept for the Cray program begins with a traditional backtrack program up to the point where the first block is computed, followed by the pregeneration of blocks 2 through 4 with additional matrices to record the compatibility between vectors within these blocks; these are repeatedly computed as the program moves through these blocks. This has the effect of determining entire columns (superchoices) at each node in the search tree, rather than a single column value one row at a time. The reason this works on the Cray to speed things up overall is because it is possible to compute many rows (vectors) of the blocks and the compatibility matrices at one time (in a single CPU cycle). If a successful block 4 is found, the program reverts to a traditional backtrack search because by this point the possible choices are so constrained that the overhead of pregeneration is greater than considering the sparse choices one at a time (the opposite issue exists before the completion of block 1 when there are too many superchoices for the computer to handle, many of which will never be used in the first place since they lack context). In any case, the previous 
explanation really does not do justice to the beautiful piece of technical work achieved to surpass the computational limits of the day [30]. As a measure of increased complexity between easy and difficult cases, the total number of completed $B 2$ s and $B 3$ s were approximately 4 billion and 155 billion respectively for each case. The article from Lam et al. is definitely worth looking at in its entirety [30]. In the end, it took an additional 2000 hours of Cray super-computer time to eliminate the remaining more complicated cases. Therefore,

$$
0=\left|P_{19}\right|=24675
$$

We must hence conclude that the assumption of the existence of a projective plane of order 10 , which has produced a flawed weight enumerator, must be false. 


\section{Implementation}

This section contains our implementation of the confirmation of the non-existence of projective plane of order 10 . Specifically, the matrix containing the polynomials (obtained from the MacWilliams identities) of the hypothetical plane of order 10's code is reduced to obtain the equation which relates the count of pertinent weight codewords. Algorithms are then presented which are developed to attempt the extension of the incidence matrix of a plane of order 10 from the staring configurations based on those pertinent codewords. Finally, the results of programs implementing the algorithms are presented. Any differences in methodology and results with previous research are contrasted as required.

\subsection{C's Weight Enumerator}

As previously indicated, for a code of length $n$, the weight enumerator is an $n^{\text {th }}$ degree polynomial homogeneous in the variables $x$ and $y$, with a coefficient $A_{j}$ of $x^{n-i} y^{j}$ equaling the number of codewords of weight $i$. We already have some information regarding the $A_{j}$ from lemmas 1.3.2, 1.3.4, 1.3.5 and 1.3.6: 
- $\mathrm{A}_{0}=1$;

- $A_{1}=A_{2}=\ldots=A_{10}=0$;

- $A_{11}=111$

- $A_{i}=0$ when $/$ is congruent to $1,2(\bmod 4)$, unknown otherwise;

- $\mathrm{A}_{i}=\mathrm{A}_{111-i}$, since the sum of all lines is the all $1 \mathrm{~s}$ codeword.

Hence, $\boldsymbol{W}_{\boldsymbol{C}}(x, y)$ is expressed as:

$$
x^{111}+11 x^{100} y^{11}+\sum_{l=0}^{21} \mathrm{~A}(4 I+12) x^{(99-4 l)} y^{(4 I+12)}+\mathrm{A}(96-4 l) x^{(96-4 I)} y^{(4 l+15)}+11 x^{11} y^{100}+y^{111}
$$

Next we want to determine $C$ 's dual code $C^{\perp}$, i.e. all vectors which are orthogonal to the codewords of $\boldsymbol{C}$ [21]. More precisely:

$$
\forall \boldsymbol{u} \in \boldsymbol{C} \text { and } \forall \boldsymbol{v} \in \boldsymbol{C}^{\perp}, \boldsymbol{u} \cdot \boldsymbol{v}=0
$$

Note that for binary vectors ${ }^{\Psi}[21]$ :

- $u \cdot v=0 \Leftrightarrow \operatorname{weight}(u * v) \mid 2$

- $u \cdot v=1 \Leftrightarrow \operatorname{weight}(u * v) \nmid 2$

\footnotetext{
$\Psi \cdot$ is the scalar product, $l$ e $x \cdot y=x_{1} y_{1}+x_{2} y_{2}++x_{n} y_{n}$,

${ }^{*}$ is the component-wise product, $t$ e $x * y=\left\langle x_{1} y_{1}, x_{2} y_{2}, \quad, x_{n} y_{n}\right\rangle$
} 
Furthermore a binary linear code consists of either all even weight codewords, or half are even and half are odd [21]. Clearly, C's $2^{56}$ codewords consist of odd and even codewords, each numbering $2^{55}$. Now we have already seen that for $\ell$ any line of the plane and $\boldsymbol{u}$ any codeword in $C,|\ell \cap u|$ is congruent to $|\boldsymbol{u}|(\bmod 2)$. Any odd weight codeword will then have a scalar product of 1 with any line, hence not orthogonal. But then this leaves the $2^{55}$ even weight codewords which are orthogonal to all lines (55 happens to be the dimension of $C^{\prime}$ s dual code $C^{\perp}$ ). Furthermore an even weight codeword is also orthogonal to all codewords in $C$, since a codeword is nothing more than a linear combination of the generators of $C$ (i.e. the lines of the plane). We must then conclude that for the $2^{55}$ even weight codewords $u_{2}$ in $C$ we obtain the following:

$$
\forall u \in C \text { and } \forall u_{2} \in C, u \cdot u_{2}=0 .
$$

Hence, $C^{\prime}$ s dual code $\boldsymbol{C}^{\perp}$ consists of all the even weight codewords of $\boldsymbol{C}$, and

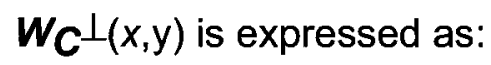

$$
x^{111}+\sum_{i=0}^{21} \mathrm{~A}(4 i+12)^{x^{(-4 i+99)} y^{(4 i+12)}+11 x^{11} y^{100}}
$$


As presented before, the MacWilliams Identities state that: If $\boldsymbol{C}$ is an $[n, n-k]$ binary linear code with dual code $C^{\perp}$, then $2^{k} \boldsymbol{W}_{\boldsymbol{C}}(x, y)=\boldsymbol{W}_{\boldsymbol{C}} \perp(x+y, x-y)$. Hence, we can write the following equality:

$$
\begin{aligned}
& \left.2^{55}\left(x^{111}+11 x^{100} y^{11}+\sum_{i=0}^{21} \mathrm{~A}(4 i+12)\right)^{(99-4 i)} y^{(4 i+12)}+\mathrm{A}(96-4 i) x^{(96-4 i)} y^{(4 i+15)}+11 x^{11} y^{100}+y^{111}\right)
\end{aligned}
$$

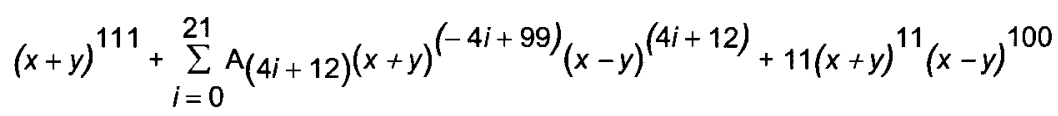

If we expand everything out and express in terms of the unknown $A_{j}$, we end up with a linear system consisting of a $23 \times 112$ matrix, where row $i=$ row $112-i$. Hence the system immediately reduces to a $23 \times 56$ matrix. Solving ${ }^{\Psi}$ this system, we find that all weights can be expressed in terms of just three parameters, which we choose as $A_{12}, A_{15}$ and $A_{16}$. To match previous notation we let $A_{19}=w_{19}$, $A_{12}=w_{12}, A_{15}=w_{15}$ and $A_{16}=w_{16}$ and express the number of weight-19 codewords as:

$$
w_{19}=24675+141 w_{12}-27 w_{15}+7 w_{16}
$$

Together with the equation that we obtained in the analysis of configurations, i.e.

\footnotetext{
${ }^{\Psi}$ See appendix B and C for details
} 


$$
w_{19}=\left|P_{19}\right|+66^{\star}\left|M_{12}\right|+8^{*}\left|M_{16}\right|
$$

we obtain the following equation, which expresses the number of primitive 19point configurations in terms of (primitive) 12, 15 and 16-point configurations:

$$
\left|P_{19}\right|=24675+75^{*}\left|M_{12}\right|-27^{*}\left|M_{15}\right|-\left|M_{16}\right|
$$

-Note that since the number $(\geq 0)$ of $M_{12}$ 's has a positive coefficient (which just increases the possible number of $\boldsymbol{P}_{19 s}$ ), we can derive the following:

$$
\left|P_{19}\right| \geq 24675-27^{*}\left|M_{15}\right|-\left|M_{16}\right|
$$

What this means in practical terms is that we need not search for the number of $M_{12}$ 's, saving us some unnecessary computation. Comparatively, it was advantageous for Lam to perform an $M_{12}$ search, since at the time the existence of a plane of order 10 was not settled and the search may have actually yielded such a plane (and hence required no further computing). But given that we "know" that no such plane exists, computing $\boldsymbol{M}_{12}$ 's would only add an extra unnecessary step to confirm non-existence. 


\subsection{Algorithmic Techniques}

So far, only high-level descriptions of the search techniques employed in previous research have been presented (e.g. backtracking, block extension, line extension). In this section a detailed presentation is given of the algorithms that are used in the development of the $\boldsymbol{M}_{15}, \boldsymbol{M}_{16}$ and $\boldsymbol{P}_{19}$ search programs implemented as part of this thesis.

The searches to be implemented can be conveniently separated into three tasks:

- find the starting configuration of heavy lines (A1 matrix);

- extend the $A 1$ to include the medium lines ( $A 2$ matrix, which include the trivially computed light lines);

- extend the $A 2$ column by column until a projective plane is found or it is no longer possible to extend without violating the properties of a projective plane. This is also described as adding blocks $(B 1, B 2, B 3$, etc...) to the A2 matrix. 


\subsection{Isomorphism Testing}

Before presenting each of the algorithms to implement the above tasks, one issue common to all three need be addressed: isomorph rejection. As discussed previously, isomorphism of partial planes must be taken into account in order to reduce the problem to a manageable time-wise computation. It is not a trivial task to determine if two partial projective planes are isomorphic. In fact, in general, any known method requires exponential time. The method that will be used here consists of expressing the partial plane as a graph, computing the certificate of its canonically-labeled isomorph [24], verifying that the computed certificate has not already been encountered and, if not, adding the certificate to a uniqueness list. If the certificate of a graph of a partial plane is already in the list, it is not processed further. This way we process only non-isomorphs.

Converting to a graph is quite trivial. Consider once again the Fano plane:

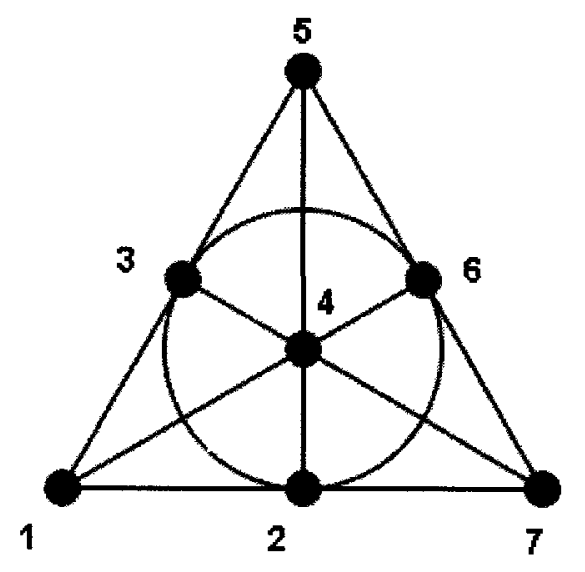

Figure 3.3.1 
In terms of a symmetric BIBD, we denote it as $D=\{\{1,2,7\},\{1,3,5\},\{1,4,6\}$, $\{2,3,6\},\{2,4,5\},\{3,4,7\},\{5,6,7\}\}$. We construct the graph $G(D)$ so that the vertices 1 to 7 correspond to the points and the vertices 8 to 14 correspond to the lines. An edge is drawn between vertices $e \in\{1,2, \ldots, 7\}$ and $f \in\{8,9, \ldots, 14\}$, if the point representing the vertex $e$ is incident with the line representing the vertex $f$. Furthermore the graph $\boldsymbol{G}(\boldsymbol{D})$ is to be bipartite, with the vertices $e \in\{1,2, \ldots, 7\}$ and $f \in\{8,9, \ldots, 14\}$ having different initial colourings, i.e. points and lines are never considered to be equivalent when computing the canonical labeling of $G(D)$. Finally, it is known that two designs $D_{1}$ and $D_{2}$ are isomorphic if and only if $G(D)_{1}$ and $G(D)_{2}$ (as coloured bipartite graphs) are isomorphic [24]. The graph $\boldsymbol{G}(\boldsymbol{D})$ equivalent to the Fano plane above follows:

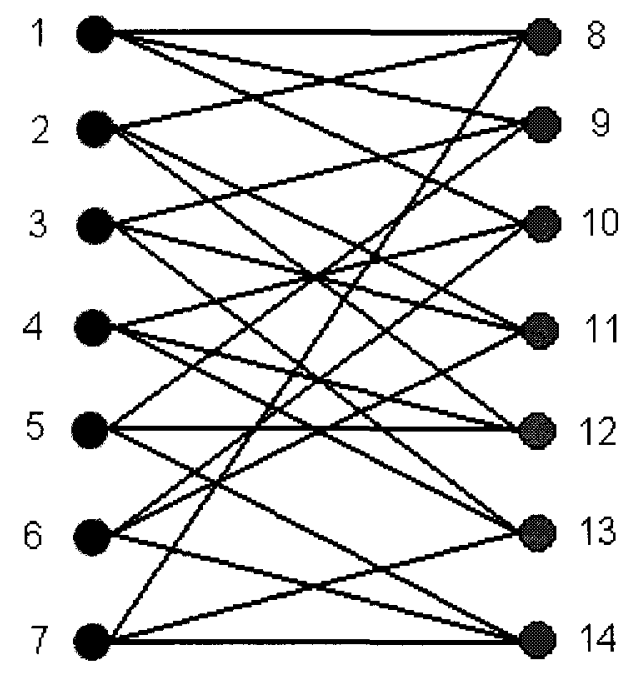

Figure 3.3.2 
Rather than implement the code to produce canonical labeling of graphs, an existing implementation is integrated into the programs. Nauty [24] (№ Automorphism, Yes?) was developed by Brendan D. McKay (a professor in Computer Science Department at the Australian National University). Its purpose is to compute automorphism groups of graphs as well as the canonical labeling and it is generally regarded as very efficient. Nauty also provides a hash value (three 64-bit integers) computed from the canonical labeling of a graph. In order to decrease memory usage, this hash value can be used as a certificate instead of the canonical labeling, although handling possible yet highly unlikely collisions ${ }^{\Psi}$ (the same hash value generated from non-isomorphs) needs to be taken into account, i.e. the actual canonical labellings are compared for equal hash values.

An important consideration is storage of certificates. Minimal searching through existing certificates is desirable to reduce computing time. Although a balanced tree algorithm would ensure a $\ln (n)$ search performance, since the search order generates the certificates in a fairly random fashion, a simple binary tree class is sufficient. For example, looking ahead to one particular search, a binary search tree with 43,624 certificates ends up with a height of 28 . In other words the longest search of length 28 is quite close to the optimal $\ln (43624)=11$ length, and much better than the expected $43624 / 2=21812$ length for a linear search.

\footnotetext{
${ }^{\Psi}$ This occurred at a rate of approximately $0.001 \%$
} 
Another consideration is when to test for isomorphism. Indeed, since isomorphism testing takes exponential time, it can't be used at every node in the search tree. In general, it should only be applied at the first few levels of a search, and any other levels where it is expected that many isomorphs will be eliminated. It takes quite a bit of analysis to determine the latter and it can vary quite a bit even for the same class of objects. Finally, there comes a point where there are just so many nodes that computing isomorphs exceeds the cost of just processing all nodes. Some details of testing levels will be further provided when the results of the searches are presented. Page 94 contains specific details about how isomorphism was applied in the implementations of the searches. 


\subsection{A1 Search}

As previously noted, the first algorithmic task is to find initial instances of heavy lines (A1 matrix) for a particular $i$-point configuration. The algorithm to determine $A 1$, follows on page 69 . It basically attempts to build the initial submatrix column by column, then row by row. The initial call is $A 1(1,1,1)$, i.e. it begins by building column 1, row 1 , starting with point 1 . The variables usedPair]][ and copies[] are initialized to all False and all zeros respectively, and keep track of which pairs and how many copies of each point are currently used. The first if statement ensures that we use no more than $i$ points. The second if statement represents that we have constructed an instance of the initial matrix so we take note of it. The first else represents that we have successfully constructed a row, so we call A1 recursively to construct the next row. Note the use of the function uniquePartialPlane() when a row is completed (i.e. testing level) to ensure that only non-isomorphic instances are considered. Finally, the last part of the algorithm attempts to add a column to the current row, verifying that a particular choice point does not already occur with one of the other points on the row (usedPair). Note that the variables endPoint, endRow and endColumn are set

relative to the particular instance being processed, e.g. for a 15-point configuration endpoint $=15$, endRow $=7$ and endColumn $=6$. This algorithm is exhaustive, i.e. it verifies all non-isomorphic possibilities. 


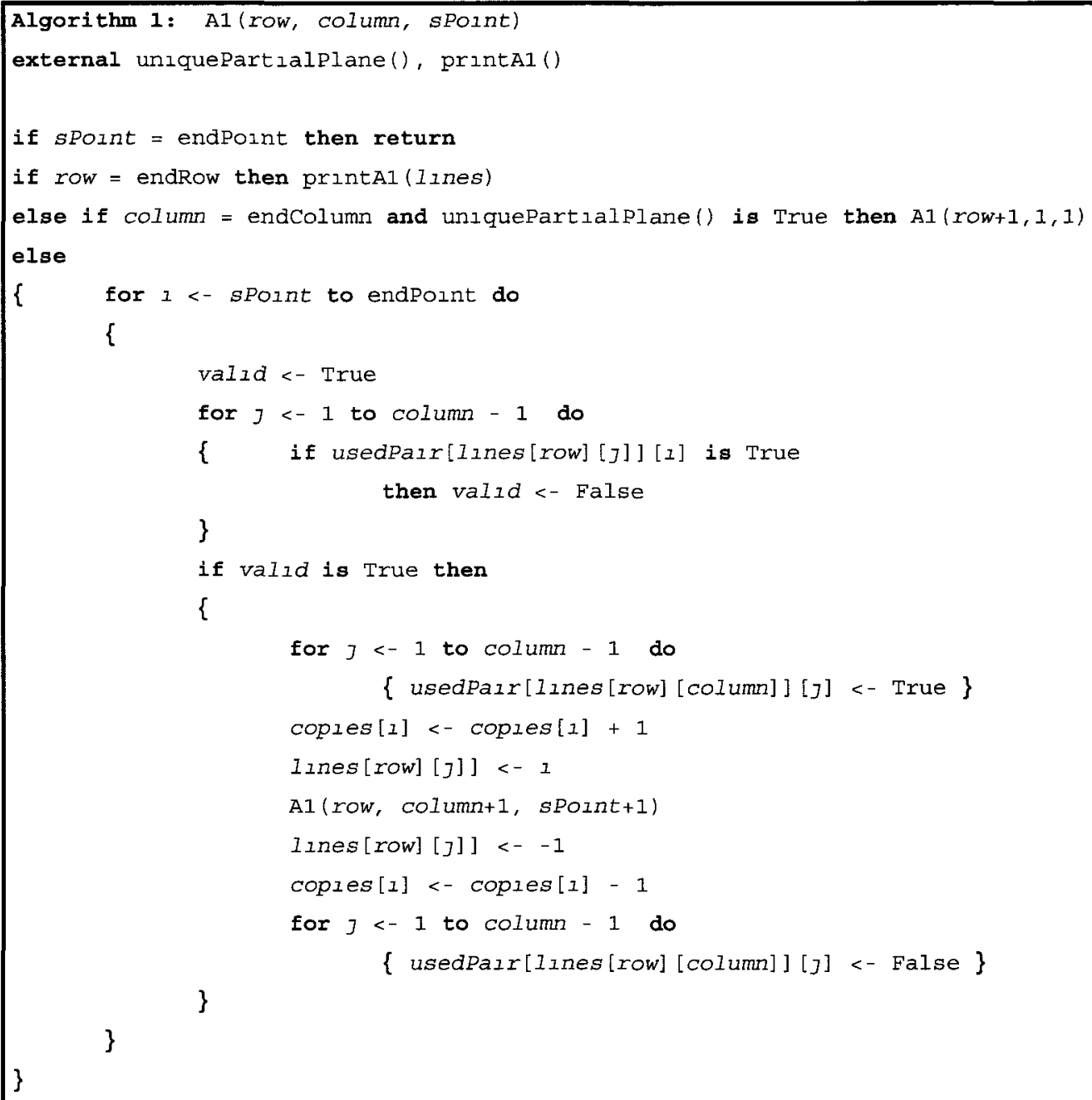

The associated programs that we have coded to do the searches are more efficient than the algorithm above in that they implement:

- an additional isomorphism of unused points;

- the use of bit operations on integers rather than arrays. 
The first of these improvements is quite simple. Only one of a set of $n$ unused points need be considered at a particular node, since all $n$ possibilities will be isomorphic. This saves processing nodes needlessly as well as determining isomorphism without calling the expensive uniquePartialPlane() function.

The second improvement relates to technical aspects of the programs. Processing of arrays using arithmetic operators is less efficient than using integers to represent the data structures and operating on these with bit manipulations (and, or, xor, shift, etc...). These are used, for example, when computing choice sets for the current node, and extracting individual bits from these choice sets. Additional details are discussed when presenting the next two algorithms, in particular the block extension which uses bit manipulation extensively. 


\subsection{A2 Search}

As previously noted, the second algorithmic task is to find instances of medium lines (A2 matrix) for a particular $i$-point configuration. The algorithm to determine $A 2$, follows on the next page. It is designed to find medium lines that contain three points, i.e. those medium lines of 15-point and 19-point configurations. While the $A 1$ search algorithm builds the matrix on a column-by-column and lineby-line basis, this algorithm builds the medium lines from the basis of a point's coincidence with other points of the $i$-point configuration. This reduces computation time versus the approach used in the $A 1$ search algorithm. Also the algorithm presented incorporates vector and bit manipulation techniques which, as stated previously, improve computation time significantly.

The central data structure in the algorithm, choiceset [point], is a vector that encodes the co-incidence of the point with all points greater than itself. For example, point 3 in a 15-point configuration co-incident with points $1,4,7$, and 9 , would have its choiceset [3] vector set to 000011010111111 . What this means is that point 3 does not yet share incidence with points $5,6,8,10,11,12$, 13, 14 and 15 (all those positions with a value of 1 ). Note that the vector indicates a 0 in position 2 , yet point 3 does not share incidence with point 2 . This is because the co-incidence of point 3 with point 2 will already have been handled (when point 2 incidences are addressed) by the time the algorithm reaches point 
3 , hence the vector need only indicate the lacking co-incidences with points greater than the point in question (in this case point 3).

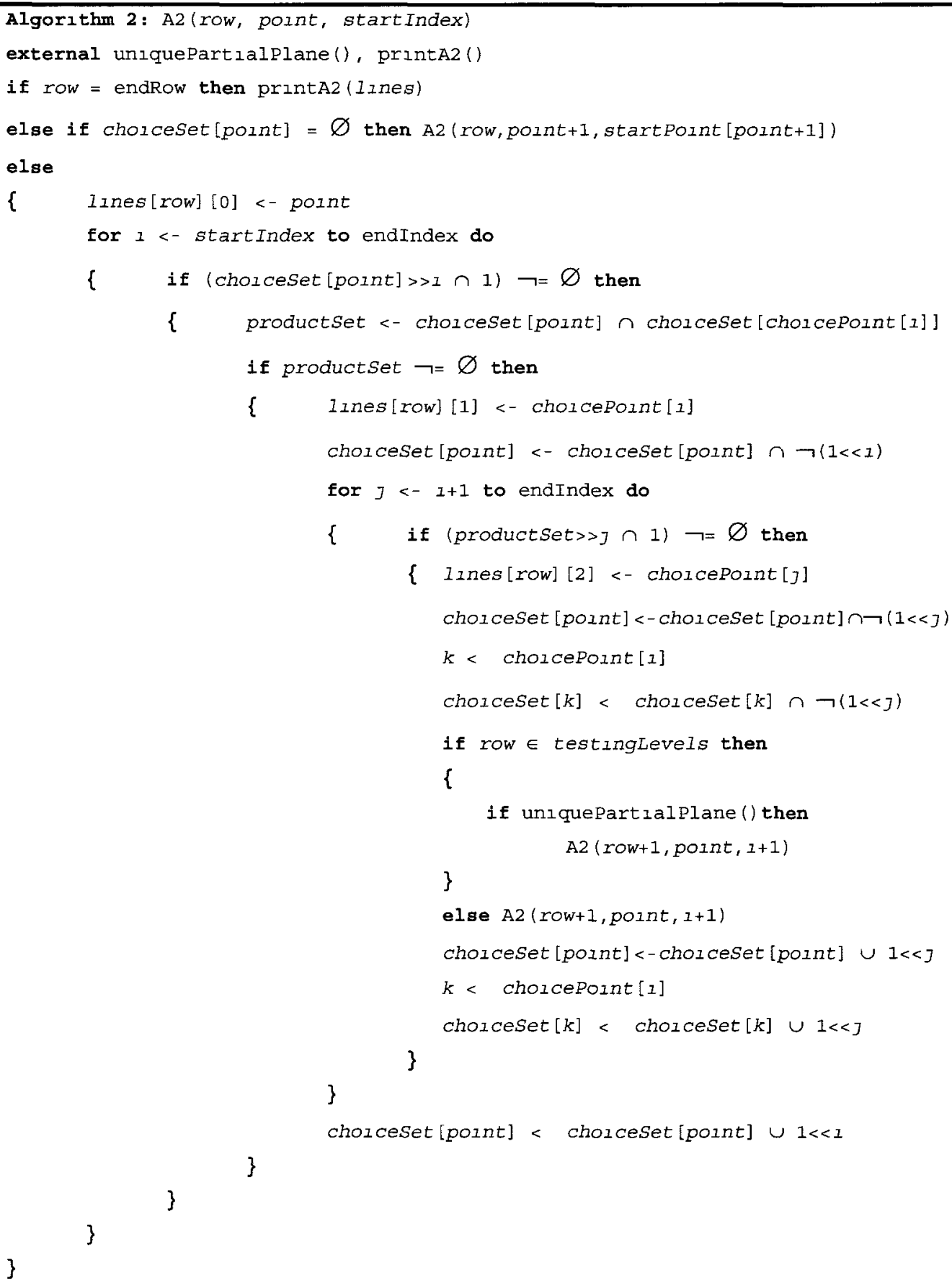


Before the initial call to $A 2(1,1,2)$, the choiceset $[i]$ vectors are initialized based on the particular $A 1$ being extended (i.e. existing incidences of the $A 1$ are encoded into the vectors). The parameters 1,1,2 mean that we begin with point 1 at row 1 with starting index 2 into point 1's choiceset [1] vector. The constants endRow and endlndex are based on the $A 1 i$-point configuration being extended, e.g. for a 15-point configuration endRow=15 and endindex=15.

The first if statement simply indicates the target number of rows has been reached, i.e. the $A 2$ has been built. The first else indicates that all incidences for the current point have been addressed, so the algorithm continues with the next point. The second else statement incorporates the work to build the current row based on the incidence requirements of the current point.

The for loop goes through the choiceset [point] vector for the current point. The if statement is entered for every position of the vector where incidence is not satisfied, i.e. the choicePoint [i]. This determination is achieved by shifting out the value of the given position to perform an AND operation, i.e. bit manipulation. Next, the intersection set (productset) of required incidences of the current point and the choicePoint [i] is determined. If the productset is not empty, all of its members, choicePoint [j] , are added in turn (the second for loop) along with the current point and choicePoint [i] to form the current 
row (and their resulting incidence vectors adjusted/de-adjusted accordingly). For each of these triples, a recursive call to $A 2($ row +1, point, $i+1)$ is invoked, which will attempt to build the next row with the current point into the next position of its incidence vector. Note that if the current row is within the prescribed isomorphism testing levels, uniquePartialPlane() will be invoked to ensure a unique isomorph before the recursive call to $\mathrm{A} 2$ in order to substantially reduce computation time. Note that a full $A 2$ is part of the testing levels so that the algorithm will generate all, but only unique, $A 2 \mathrm{~s}$.

Note that unlike the $A 1$ search algorithm, this algorithm is very close to its actual associated program. The only real difference is that the program uses more efficient decision evaluations, unlike the algorithm's set operations (i.e. $\cap, \cup, \in$ , Ø). Also, a particular point's starting index into its associated incidence vector is determined based on the actual incidences of the input $A 1$, i.e. the vector's first non-zero position > point, rather than simply the vector's position right after the point value (which avoids unnecessary looping).

\subsection{Block Search}

As previously noted, the last algorithmic task is to find instances of complete columns, i.e. blocks ( $\mathrm{Bi}$ matrices) for a particular i-point configuration. Recall the method of extending the matrix relative to a block, referencing the following 
diagram (an $A 2$ without most medium lines incident with points 1 through 5 , nor light lines):

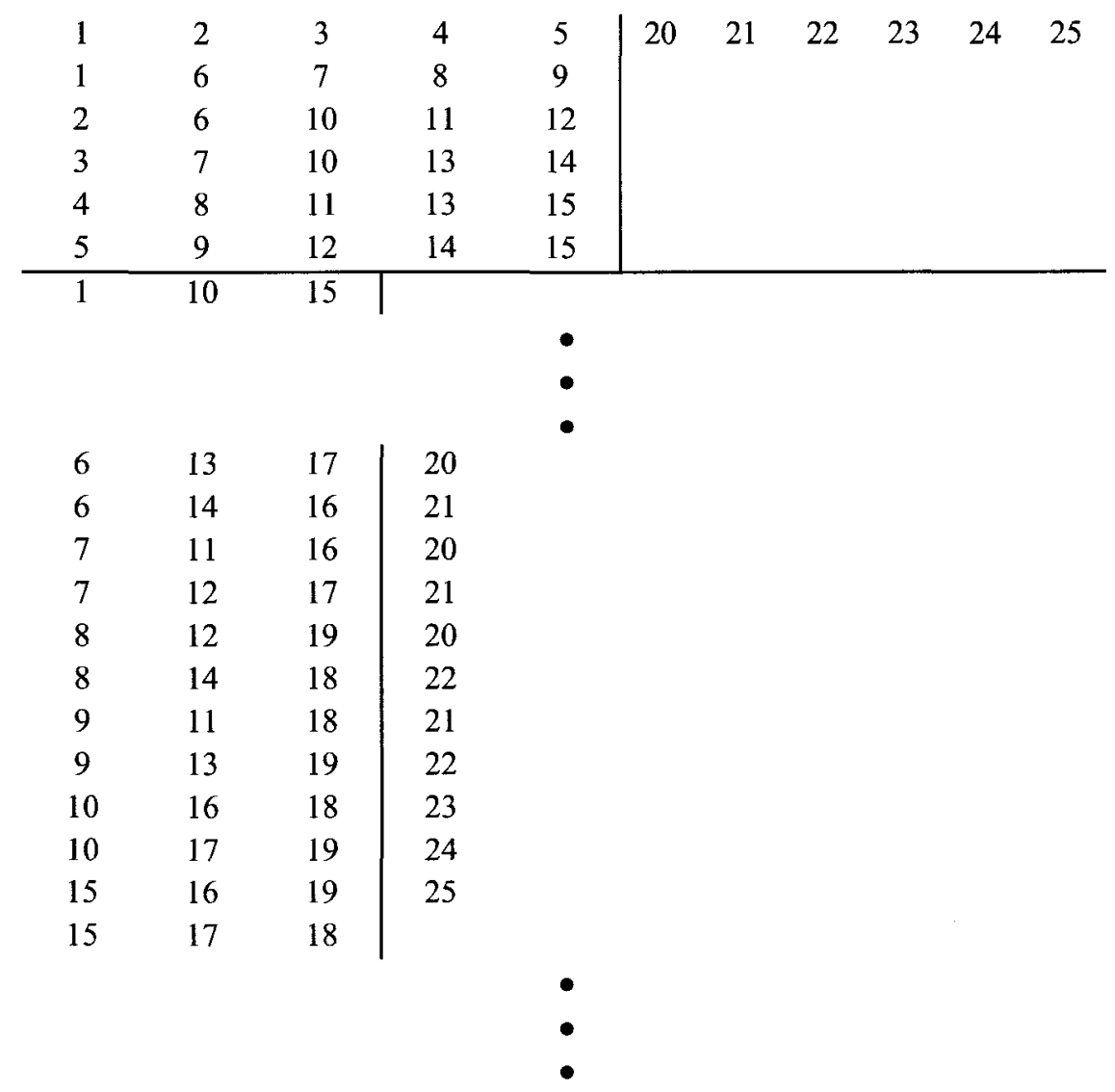

The first block is line 1 , to which the 6 required points to complete the line are added. Then, the medium lines are addressed in terms of incidence with the added points (note that no medium line containing points 1 through 5 can contain any of the block-1 points due to the constraints of line 1). Finally, the light lines (again, other than those with points 1 through 5) are addressed. A completed block is one where block-1 point incidences have been addressed relative to all 
rows in the matrix, at which point the addition of another block would be attempted.

The algorithm to extend the matrix by adding blocks follows (note that the notation $\{\mathrm{i}\}$ indicates that the algorithm is working on the $\mathrm{i}^{\text {th }}$ block):

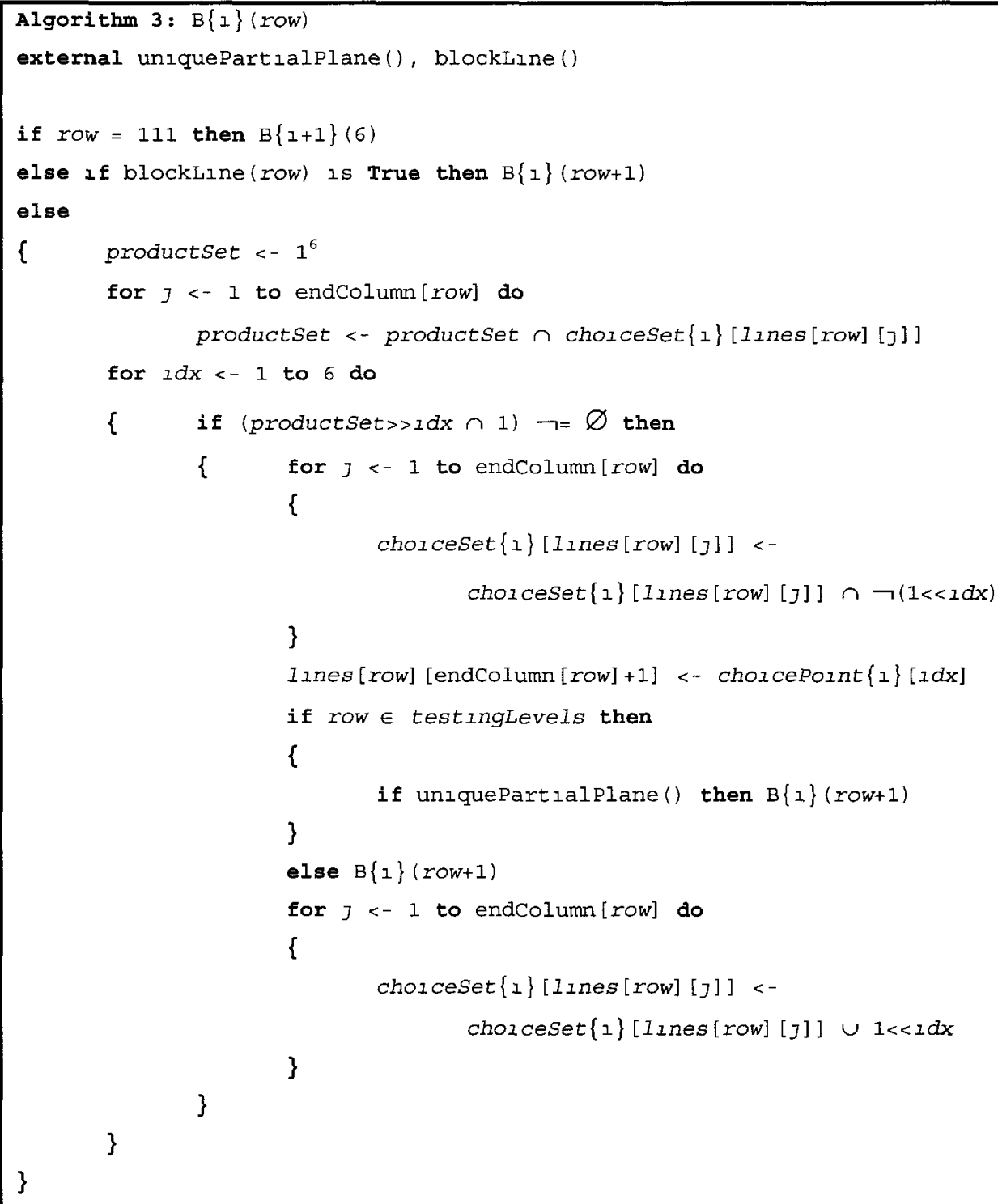


Once again the central data structure in the algorithm, choiceSet $\{i\}$ [point], is a vector that indicates the incidence of the point, but with the points of the block. Before the initial call to $B\{i\}(1)$, the choiceSet $\{i\}[]$ vectors are initialized to 111111 . The parameter 1 means that we begin at row 1 for block \{i\}.

The first if statement simply indicates the target number of rows has been reached (the $B\{i\}$ has been built) and a call to $B\{i+1\}(1)$ is invoked, i.e. the algorithm will attempt to extend the matrix relative to the next block. The first else indicates that the current row is incident with the block row, so the algorithm continues to the next row. The second else statement incorporates the work to add choice points from the block to the current row.

The first for loop computes the intersection set (productset) of incidences remaining of the points of the current row (with the points of the block). The second for loop iterates through the productset vector, determining the valid incidences of the current row against the block set (bit values of 1). For each of these, the choiceSet $\{i\}$ [] vector of the points on the row are adjusted - and accordingly de-adjusted at the end of the loop - against the particular block point. $A$ recursive call to $B\{i\}($ row +1$)$ is invoked, which will attempt to add a column (a point from the block set) to the next row. Note that if the current row is within the prescribed isomorphism testing levels, uniquePartialPlane() will be 
invoked to ensure a unique isomorph before the recursive call to $\mathrm{B}\{\mathrm{i}\}$ in order to substantially reduce computation time.

While this algorithm is fairly simple, most of the computation for the search of a projective plane of order 10 will involve these block extensions. Hence, the programs expressing this algorithm have to be as efficient as possible. As with the $A 2$ search algorithm, this algorithm is similar to its actual associated programs. One difference is that the programs use more efficient decision evaluations, unlike the algorithm's set operations (i.e. $\cap, \cup, \in, \varnothing)$.

In addition, the block extension programs exhibit 3 major differences as follows (listed in order of added computational efficiency):

- isomorphism of light lines;

- improved processing of productset vector;

- dynamic row order block extension to maximize constraints.

The first computational improvement, isomorphism of light lines, can be applied in the first few block extensions of the search depending on the specific A2. Recall the following example, now with some light lines appearing: 


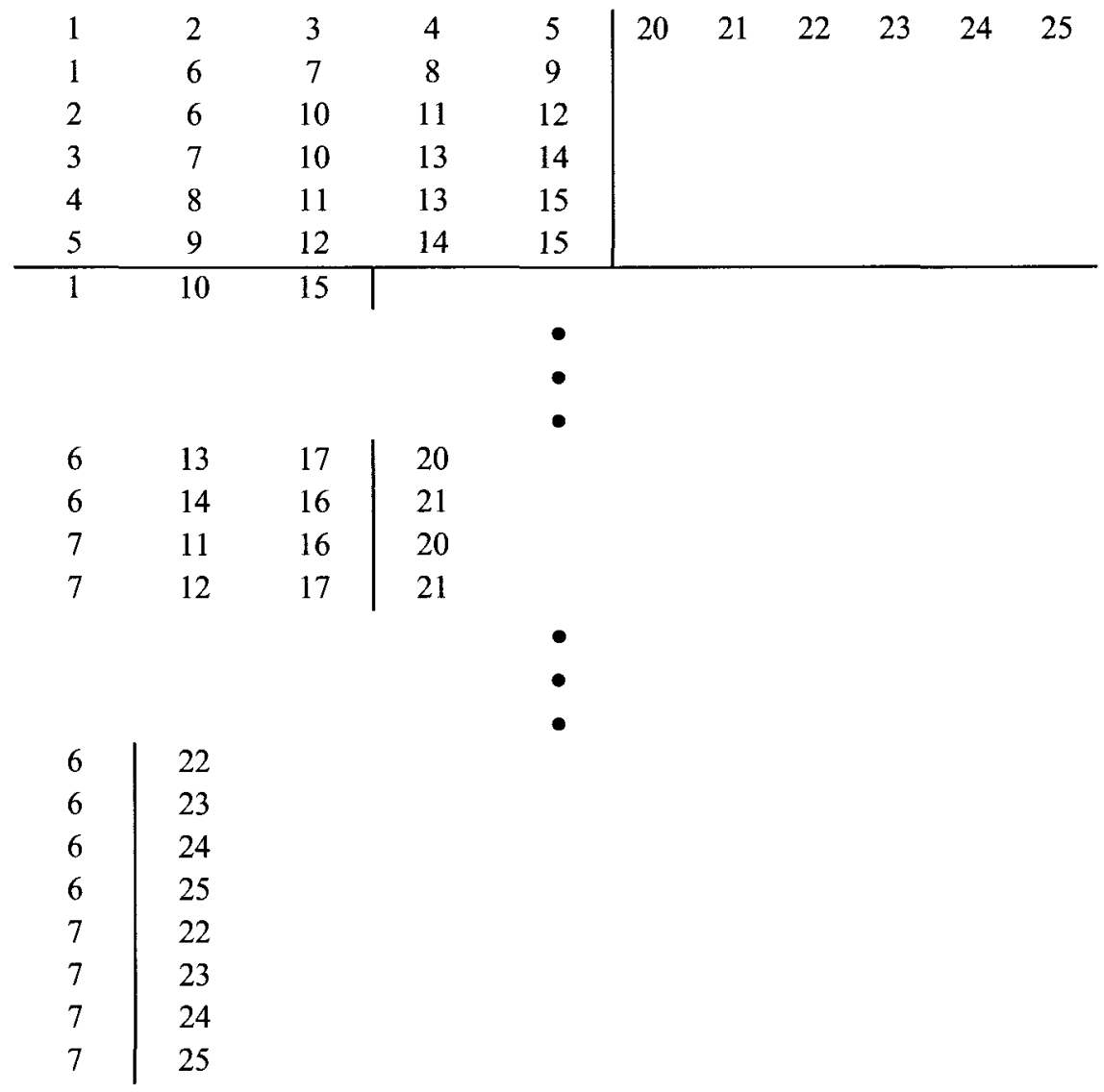

The algorithm, as presented, would try all remaining block point possibilities when reaching the light lines, i.e. in this case there are actually 4 possibilities for the $1^{\text {st }}$ light line containing point 6 , then 3 possibilities for the $2^{\text {nd }}$ light line containing point 6 , etc... in fact 4 ! different possibilities for the set of light lines containing point 6 , when really there is only one possibility up to isomorphism. This occurs for all sets of light lines, partitioned by point. It is of course possible to eliminate these redundancies through the isomorphism check, but at a high cost given that we already know that the sets of light lines are isomorphic relative to the block 
points. Hence, the actual programs consider only one light line configuration automatically, based on how the medium lines are built. This saves considerable time at the beginning of a block extension search.

The next computational enhancement, improving the processing of the productset vector, benefits the programs every time a column is added. The vector consists of six bit positions, hence there are $2^{6}=64$ possible values for a vector. These are pre-computed and a set of arrays are used to indicate which positions of a given vector value actually have their bits set to a 1 . Therefore instead of iterating through a vector 6 times to find the 1-bits, we only iterate $n$ times, where $n$ is the actual number of 1-bits in the vector. This avoids unnecessary looping and saves considerable time since vector processing is done at every node in the search tree.

The final computational improvement, dynamic row order block extension to maximize constraints, once again benefits the programs every time a column is added. The rows of the matrix are not well balanced point-wise, especially in the latter block extensions. This means that some points have higher incidence counts than other points, i.e. they occur on lines with higher point counts. If the rows of these points are addressed first when performing the block extensions, there will be fewer resulting search tree nodes since the constraints will be greater from the beginning. Implementing this requires that an ordering of the 
processing of the lines be computed (only) once for each block for a given $A 2$. This applies to every block after the first, and has a significant cumulative effect.

In terms of cost, these three improvements are quite cheap. The arrays used in the improved processing of productset vector technique need only be populated at the beginning of a run. The other two techniques' arrays need only be generated on the first instance of a $B i$ within each $A 2$.

\subsection{Search Instances}

This section discusses the results of the $\boldsymbol{M}_{\mathbf{1 5}}, \boldsymbol{M}_{\mathbf{1 6}}$ and $\boldsymbol{P}_{19}$ searches (detailed numbers are contained in the appendix). Note that all programs used incorporate the algorithmic techniques previously discussed, and were executed on 4 gigabytes RAM, AMD cpu $(2.4 \mathrm{gHz})$ multi-core computers running 64 bit Linux operating systems.

\section{M15 Search}

As previously noted and confirmed by current programs developed, the single $A 2$ starting configuration for an $M_{15}$ is the following: 


\begin{tabular}{ccccc|}
1 & 2 & 3 & 4 & 5 \\
1 & 6 & 7 & 8 & 9 \\
2 & 6 & 10 & 11 & 12 \\
3 & 7 & 10 & 13 & 14 \\
4 & 8 & 11 & 13 & 15 \\
5 & 9 & 12 & 14 & 15 \\
\hline 1 & 10 & 15 & & \\
1 & 11 & 14 & & \\
1 & 12 & 13 & & \\
2 & 7 & 15 & & \\
2 & 8 & 14 & & \\
2 & 9 & 13 & & \\
3 & 6 & 15 & & \\
3 & 8 & 12 & & \\
3 & 9 & 11 & & \\
4 & 6 & 14 & & \\
4 & 7 & 12 & \\
4 & 9 & 10 & & \\
5 & 6 & 13 & & \\
5 & 7 & 11 & & \\
5 & 8 & 10 & &
\end{tabular}

Also unique are the 90 light lines, partitioned into 15 groups of six lines on which lie the points of the $\boldsymbol{M}_{\mathbf{1 5}}$. The program computes that there is only one instance, up to isomorphism, of the extended matrix relative to block 1 (i.e. there is only one B1). The program then finds $79 \mathrm{~B} 2 \mathrm{~s}$ and $124,126,848 \mathrm{~B} 3 \mathrm{~s}$, but cannot extend any of the latter to a B4. Note that full isomorphism was used up to block 2 and for the first 10 rows of the $3^{\text {rd }}$ block.

An interesting aspect of the present implementation is the block based approach used, rather than the line based search performed by MacWilliams et al. While the fact that the 2 minute running time of this search compared to the 3 hours required by MacWilliams et al. can in part be explained due to computer speed, 
the other major contributing factor is the block vs. line based method. In fact, a verification of the existence of $\boldsymbol{M}_{15}$ s was performed in and implemented as a line based search [25]. The number of search nodes required was about $3 \times 10^{9}$ while the present search required only $2 \times 10^{8}$, a significant difference indicating the superior efficiency of the block based method for this type of search.

This search determined that no $M_{15}$ s exist and consequently that only $M_{16}$ s of primitive type may exist, respectively, in a projective plane of order 10 . Hence, the following may be concluded on this basis:

$$
\left|M_{15}\right|=0 \rightarrow\left|P_{19}\right| \geq 24675-\left|M_{16}\right|
$$

\section{M16 Search}

While previous researchers (Carter and Lam et al. $[6,26]$ ) performed their searches directly on the starting configurations and incidence matrix form imposed by a 16-point configuration, it was decided to implement a search of $16-$ type $M_{19}$ s. Recall from the detailed analysis of configurations, that an $M_{16}$ exists in the plane if and only if a 16 -type $\boldsymbol{M}_{19}$ exists. There are two main (related) reasons to proceed in this fashion: 
- Multiple specialized programs were necessary to search for $\mathbf{M}_{\mathbf{1 6}} \mathbf{S}$;

- The same core program can be used to search for $\boldsymbol{M}_{\mathbf{1 5}} \mathbf{s}, \boldsymbol{P}_{19}$ and 16-type $M_{19 s}$ with very few changes.

The cost of having a general core for all three searches is decreased computational efficiency, particularly in the case of the $\boldsymbol{M}_{16}$ search. However given the remarkably improved speed of today's computers, it is worth trading some efficiency in order to reduce the likelihood of bugs by having a single core program.

As before, the first task is to find the possible starting configurations. Recall that a 16-type $\boldsymbol{M}_{19}$ consists of 19 points on four lines, one of these with seven points and the other three lines with 5 points. A program based on algorithm 1 found that there are 22 possibilities up to isomorphism. However, four of these cases can be immediately dismissed just by examining the matrix in question. One example is presented below. On the heavy lines, point 1 shares incidence with all points from 2 to 15 . Therefore, its incidence with points 16 to 19 must be addressed on the triple (medium) lines. However, points 16 to 19 already appear on the same line. Hence it is impossible to combine them in pairs along with point 1 to form the required triple lines and satisfy the incidence requirements. 


$\begin{array}{ccccccc}1 & 2 & 3 & 4 & 5 & 6 & 7 \\ 1 & 8 & 9 & 10 & 11 & & \\ 1 & 12 & 13 & 14 & 15 & & \\ 2 & 16 & 17 & 18 & 19 & & \end{array}$

The next task is to add 40 triple lines which will address the remaining incidence requirements of the 19 points among themselves. 12 more cases are eliminated because it is impossible to add these 40 lines without encountering a conflict. One of these cases is presented below:

$\begin{array}{ccccccc}1 & 2 & 3 & 4 & 5 & 6 & 7 \\ 1 & 8 & 9 & 10 & 11 & & \\ 2 & 8 & 12 & 13 & 14 & & \\ 8 & 15 & 16 & 17 & 18 & & \end{array}$

When one examines this matrix, it is not obvious that it cannot be extended to include the 40 triple lines as with the matrix on the previous page. However the program based on algorithm 2 determines that of the 8,827 possibilities for the $23^{\text {rd }}$ line, none can be extended to a $24^{\text {th }}$ line without conflict.

So we are left with only 6 cases $^{\Psi}$ of the original 22 , which are presented on the following page. The ones on the left are the most difficult cases, since they have only inside intersections (Lam's terminology, signifying that all lines intersect within the 19 points of the configuration), while the ones on the right have some outside intersections. Altogether, the implemented program extends the 6 cases to 246,828 A2s.

\footnotetext{
${ }^{\Psi}$ Is it a coincidence that there are also 6 starting configurations for an $\boldsymbol{M}_{\mathbf{1 6}}$ ?
} 


$\begin{array}{cccccccccccccc}1 & 2 & 3 & 4 & 5 & 6 & 7 & 1 & 2 & 3 & 4 & 5 & 6 & 7 \\ 1 & 8 & 9 & 10 & 11 & & & 1 & 8 & 9 & 10 & 11 & & \\ 1 & 12 & 13 & 14 & 15 & & & 2 & 8 & 12 & 13 & 14 & & \\ 1 & 16 & 17 & 18 & 19 & & & 3 & 9 & 15 & 16 & 17 & & \\ & & & & & & & & & & & & & \\ 1 & 2 & 3 & 4 & 5 & 6 & 7 & 1 & 2 & 3 & 4 & 5 & 6 & 7 \\ 1 & 8 & 9 & 10 & 11 & & & 1 & 8 & 9 & 10 & 11 & & \\ 1 & 12 & 13 & 14 & 15 & & & 2 & 8 & 12 & 13 & 14 & & \\ 2 & 8 & 12 & 16 & 17 & & & 3 & 15 & 16 & 17 & 18 & & \\ & & & & & & & & & & & & & \\ 1 & 2 & 3 & 4 & 5 & 6 & 7 & 1 & 2 & 3 & 4 & 5 & 6 & 7 \\ 1 & 8 & 9 & 10 & 11 & & & 1 & 8 & 9 & 10 & 11 & & \\ 2 & 8 & 12 & 13 & 14 & & & 2 & 12 & 13 & 14 & 15 & & \\ 3 & 9 & 12 & 15 & 16 & & & 3 & 16 & 17 & 18 & 19 & & \end{array}$

The final task is to extend the $A 2 \mathrm{~s}+$ light lines relative to the 4 blocks defined by the heavy lines. As pointed out above, the outside intersection cases must be handled differently than the inside intersection cases. As presented in the chronology of the solution, Lam et al. deal with the outside intersection blocks as early as possible in the extension of the matrix, which leads to less computation. However, the current core program is more readily adapted to the opposite approach. For example, the block extension of this particular case is handled in the following manner:

$$
\begin{array}{ccccc|cc|ccccc}
1 & 2 & 3 & 4 & 5 & 6 & 7 & 20 & 21 & 22 & 23 & \text { Block 1 } \\
1 & 8 & 9 & 10 & 11 & 24 & 25 & 26 & 27 & 28 & 29 & \text { Block 2 } \\
2 & 8 & 12 & 13 & 14 & 30 & 31 & 32 & 33 & 34 & 35 & \text { Block 3 } \\
3 & 9 & 15 & 16 & 17 & \mathbf{3 0} & 36 & 37 & 38 & 39 & 40 & \text { Block 4 }
\end{array}
$$


Note that Lam's method would have processed blocks 3 and 4 first, however it was hoped (perhaps naively) that the decrease in computational speed afforded is not as crucial due to the technology available today.

Note that since there are only four heavy lines, for any successfully built B4s one of the rows with the most points is chosen to become the $5^{\text {th }}$ block. In all cases, no $B 4$ could be extended to a full plane.

The difficulty of the first case should be highlighted. It is the reason that our $M_{16}$ search took much longer than Carter and Lam's search. Using the current program, all $A 2 s$ for this case extend to approximately $10^{10}$ partial incidence matrices by the time block 3 is completed. Although few of these extend to a fourth block, the program requires about $2 / 3$ seconds to process each of these $10^{10}$ cases. This equates to about 300 years of non-stop processing on the current hardware (available for the purpose of this thesis).

In order to reduce the amount of computation necessary, a trick (one of many used by Lam et al. on the difficult $M_{16}$ s which could not be handled by Carter) is necessary [26]. First off, the block order is processed as follows:

\begin{tabular}{ccccc|ccccccc}
1 & 8 & 9 & 10 & 11 & 20 & 21 & 22 & 23 & 24 & 25 & Block 1 \\
1 & 12 & 13 & 14 & 15 & 26 & 27 & 28 & 29 & 30 & 31 & Block 2 \\
1 & 16 & 17 & 18 & 19 & 32 & 33 & 34 & 35 & 36 & 37 & Block 3 \\
1 & 2 & 3 & 4 & 5 & 6 & 7 & 38 & 39 & 40 & 41 & Block 4
\end{tabular}


The idea is then to separately pregenerate the incidences relative to block 1 , block 2 and block 3 , and order them ascending by their certificates. The program will process the first $B 1$ against all possible $B 2 s$ and $B 3$ s (assemble all three into a single compatible incidence structure), and continue the more traditional extension of each to subsequent blocks if possible. On the next $B 1$, any $B 2$ s and $B 3$ s that are isomorphic to the first $B 1$ need not be considered. Continuing in this manner, each $B 1$ processed eliminates more and more $B 2 s$ and $B 3 s$ in the subsequent cycles. Considerable time is saved mainly due to the fact that less redundant isomorphs are processed without the expensive cost of isomorphism testing at deep levels of the search tree. Also, pregeneration of the Bis avoids repetitious building and tearing down of the incidence matrix again and again and again (the cost of assembly, while not negligible, is less than that of pure backtracking). It is, however, quite tricky to implement, but in this case it is necessary and really only possible due to the symmetry exhibited by the starting structure.

The second case (middle left on page 86), while not as complex as the first, is also quite time-consuming. If the following block ordering is used, it would take approximately 50 years to process the near $10^{13} \mathrm{~B} 3 \mathrm{~s}$ on the current hardware:

\begin{tabular}{ccccc|cc|ccccc}
1 & 2 & 3 & 4 & 5 & 6 & 7 & 20 & 21 & 22 & 23 & Block 1 \\
1 & 8 & 9 & 10 & 11 & 24 & 25 & 26 & 27 & 28 & 29 & Block 2 \\
1 & 12 & 13 & 14 & 15 & 30 & 31 & 32 & 33 & 34 & 35 & Block 3 \\
2 & 8 & 12 & 16 & 17 & 36 & 37 & 38 & 39 & 40 & 41 & Block 4
\end{tabular}


The solution for this case is quite simple, we permute the rows in the following manner to change the block order:

\begin{tabular}{ccccccc|ccccc}
1 & 2 & 3 & 4 & 5 & 6 & 7 & 20 & 21 & 22 & 23 & Block 1 \\
2 & 8 & 12 & 16 & 17 & 24 & 25 & 26 & 27 & 28 & 29 & Block 2 \\
1 & 12 & 13 & 14 & 15 & 30 & 31 & 32 & 33 & 34 & 35 & Block 3 \\
1 & 8 & 9 & 10 & 11 & 36 & 37 & 38 & 39 & 40 & 41 & Block 4
\end{tabular}

This block ordering, coupled with the "dynamic row order block extension to maximize constraints" optimization presented in the algorithm section, reduces the computing time to a manageable level. The reason is that the program now need only process $10^{10} B 3 \mathrm{~s}^{\Psi}$, as well as generates fewer nodes in the search tree. In fact this combination, used on the present case, will be crucial in the execution of the $\boldsymbol{P}_{19}$ search within a reasonable timeframe.

In the end, the number of search nodes required for all 6 cases was about $10^{15}$, and the program based on algorithm 3 determined that no 16-type $M_{19}$ s, and consequently, no $M_{16}$ s of primitive type exist in a projective plane of order 10 . Hence, the following may be concluded on this basis:

$$
\left|M_{16}\right|=0 \rightarrow\left|P_{19}\right| \geq 24675
$$

Before moving on to the $\boldsymbol{P}_{19}$ search, a few words should be said about the testing levels for isomorphism, as per the following:

\footnotetext{
${ }^{\Psi}$ In both cases the $B 3 \mathrm{~s}$ are not unique up to isomorphism, with a lower redundancy in the latter.
} 
- Completed $A 1 \mathrm{~s}$ and $A 2 \mathrm{~s}$ are unique up to isomorphism in all searches;

- Bis (>B1) are tested for isomorphism only at early points in the search tree (for example, all Bis are tested at least up to the $4^{\text {th }}$ row).

The first statement is fairly straightforward. Isomorphism testing is always done at every completion of one of these objects. In addition, testing for A2's is turned off when the ratio of rejected isomorphs vs total isomorphs falls below $90 \%$, and turned back on in the last three rows.

The second statement requires a bit more clarification in that the isomorphism testing applied is not accomplished with the NAUTY integration. Rather it is an isomorphism check similar to the isomorphism of light lines technique, performed in conjunction with the dynamic row order technique, as described in the algorithm section. Specifically, in addition to determining the point with the most incidences, all (admissible) rows containing this specific point are placed at the beginning of the ordering, i.e. the block is extended relative to these rows all containing the given point first. Thus, up to isomorphism, there is only one way to extend the block relative to these first few rows (between 4 and 6, depending on inside/outside intersections and the remaining light lines through the specific point). This allows a substantial number of redundant instances to be discarded without the expensive cost of the NAUTY isomorphism testing, which at the $B \mathrm{i}$ levels would take significantly more time than no testing at all ${ }^{\Psi}$.

\footnotetext{
${ }^{\Psi}$ Note that without this theoretic reduction, the amount of computation would be unreasonably high.
} 


\section{P19 Search}

The implication at the bottom of page 89 is significant: for a projective plane of order 10 to exist, there must exist at least $24,675 \boldsymbol{P}_{19}$ s. In other words, we must be able to build the incidence matrix from the starting configurations imposed by primitive codewords of weight 19: if we cannot build it in this manner, then there does not exist a projective plane of order 10 .

As before, the first task is to find the possible starting configurations. Recall that a $\boldsymbol{P}_{19}$ consists of 19 points on 6 lines, all with 5 points. A program based on algorithm 1 found that there are 66 possibilities up to isomorphism, which agrees with previous research $[3,7,27]$. However, 12 of these cases can be immediately dismissed just by examining the matrix in question. For example [27], on the following matrix point 1 does not yet share incidence with points 18 and 19 . It must address this on a single line, but points 18 and 19 already appear together on line 6 . It is impossible to complete the required triple lines and satisfy the incidence requirements. In total 12 cases are eliminated this way, and the program confirms that two more, less obvious, cases cannot be completed to the triple lines.

$\begin{array}{ccccc}1 & 2 & 3 & 4 & 5 \\ 1 & 6 & 7 & 8 & 9 \\ 1 & 10 & 11 & 12 & 13 \\ 1 & 14 & 15 & 16 & 17 \\ 2 & 6 & 10 & 14 & 18 \\ 3 & 7 & 11 & 18 & 19\end{array}$


Another 4 cases are eliminated because they imply the existence of an $\boldsymbol{M}_{16}$ [27]. As with the following example, there exist 3 lines which are mutually nonintersecting. If one adds these lines to the $\boldsymbol{P}_{19}$, the sum is a codeword of weight 16 which was determined not to exist. Specifically, the sum of lines 4,5 and 6 together with the $\boldsymbol{P}_{19}$ yields a codeword with points $<1,5,9,13,40,41,42,43$, $45,46,47,48,49,50,51,52>$, i.e. a codeword of weight 16 .

$\begin{array}{ccccccccccc}1 & 2 & 3 & 4 & 5 & 20 & 21 & 22 & 23 & 24 & 25 \\ 1 & 6 & 7 & 8 & 9 & 26 & 27 & 28 & 29 & 30 & 31 \\ 1 & 10 & 11 & 12 & 13 & 32 & 33 & 34 & 35 & 36 & 37 \\ 2 & 6 & 10 & 14 & 15 & 38 & 39 & 40 & 41 & 42 & 43 \\ 3 & 7 & 11 & 16 & 17 & 38 & 44 & 45 & 46 & 47 & 48 \\ 4 & 8 & 12 & 18 & 19 & 39 & 44 & 49 & 50 & 51 & 52\end{array}$

It should be noted that rows 4,5 and 6 cannot intersect in a common outside point, since it can be established that an outside point cannot be on more than 2 heavy lines [27].

Although Lam eliminates a few more cases with ad-hoc arguments, we will process the remaining 48 cases. The next task is to generate the $A 2 s$ for these 48 cases. The implemented program extends these 48 cases to approximately 650,000 A2s.

Note that the results of the considerably longer $\boldsymbol{P}_{19}$ search can be more readily contrasted to Lam's search since the techniques used are similar, particularly with 
regard to this $A 2$ search $[3,7]$. The present $A 2$ counts (unique up to isomorphism) agree with Lam's results in all but 7 cases. In all but one of these cases, the present counts are less than Lam's numbers. After discussing this issue with Professor Clement Lam himself and following an independent verification which he performed, the present numbers were confirmed as the correct counts, unique up to isomorphism. It is likely that Lam's numbers included some redundant cases.

This leaves the case where the present count (also confirmed as correct) is greater than Lam's result. This one is more interesting since it means that some A2s were not accounted for ${ }^{\Psi}$ in the original search performed by Lam over 20 years ago, and hence could lead to the construction of a plane of order 10. Given the title of this thesis however, it is easy to conclude that these additional $A 2 \mathrm{~s}$ unfortunately did not lead to such a result.

As before, the final task is to extend the $A 2 s+$ light lines relative to the 6 blocks defined by the heavy lines. Once again, the outside intersection cases must be handled differently than the inside intersection cases. Unlike in the $\boldsymbol{M}_{\mathbf{1 6}}$ case, since the $\boldsymbol{P}_{19}$ search is significantly greater, the outside intersection cases are handled in the same way as Lam did, i.e. the blocks with outside intersections are addressed by the program as early as possible to reduce the computation time.

\footnotetext{
${ }^{\Psi}$ It is hopefully not too bold to suggest that this single discrepancy further justifies the merit in the undertaking of this thesis
} 
The $B \mathrm{i}$ counts obtained by the current program for the $A 1$ s with outside intersections are very similar to the numbers obtained by $\operatorname{Lam}^{\Psi}[7]$, i.e. the relative orders of magnitude between the different $\mathrm{Bi}$ are the same. The differences in actual numbers can be accounted for by the fact that neither the present Bi numbers nor Lam's were unique up to isomorphism. The counts with the least difference occur on the B4s. This is not unusual since they are constructed by extended the surviving $B 3 \mathrm{~s}$, which are themselves severely constrained and hence do not result in many $B 4 \mathrm{~s}$.

As with the outside intersection cases, the differences in actual numbers for the inside only intersections can be partially accounted for by the fact that neither the present Bi numbers nor Lam's were unique up to isomorphism [7]. In addition, the different algorithms used for these inside (only) intersection cases likely also has a significant impact. Finally, the block order also makes a big difference.

As discussed in the chronology section, Lam used a different type of program on a super computer to solve the inside (only) intersection cases. The present solution uses pure backtracking, i.e. the same core program as with all other searches. It is in this instance that the combination of four inexpensive search enhancements have the most impact, i.e. isomorphism of light lines, optimal block ordering plus dynamic row order block extension to maximize constraints and

\footnotetext{
${ }^{\Psi}$ The greatest vanance is with the 7 cases where the $A 2$ counts are different
} 
theoretic reduction of redundant configurations in the first few lines of blocks 2 through 4. The following table compares the orders of magnitude for the Bi total counts for Lam's and the present search on inside (only) intersections:

\begin{tabular}{|c|c|c|}
\hline & Lam & Roy \\
\hline$B 1$ & $10^{7}$ & $10^{7}$ \\
\hline$B 2$ & $10^{11}$ & $10^{11}$ \\
\hline$B 3$ & $10^{10}$ & $10^{9}$ \\
\hline$B 4$ & $10^{6}$ & $10^{4}$ \\
\hline
\end{tabular}

In the end, the number of search nodes required for all $\boldsymbol{P}_{19}$ cases was about $10^{15}$, and the program based on algorithm 3 determined that none could be extended to full incidence matrix of a projective plane of order 10 . Hence, the following may be concluded on this basis:

$$
\left|P_{19}\right|=0
$$

However we have already demonstrated that

$$
\left|P_{19}\right| \geq 24675
$$


Once again, we must sadly conclude that the assumption of the existence of a projective plane of order 10 , which has produced a flawed weight enumerator, must be false. 


\section{Conclusion}

Another way to interpret this result is that, by taking into account the restrictions imposed on the incidence matrix by the configurations induced by the associated error-correcting code, we should have been able to construct a projective plane of order 10 had one actually existed. Our search, while in vain, has benefited in the substantial reduction of both the possible starting configurations of the incidence matrix and the number of possible branches at each partial solution point in the search tree afforded by the analysis of the associated, and in this case, ironically non-existent error-correcting code. While it would have been nicer to actually find a projective plane of order 10 , it is nonetheless satisfying to confirm its nonexistence.

Some final words should be said about the "correctness" of this result. As Lam mentions, there are potentially two kinds of errors that may arise: software bugs and hardware failures [3]. The former is where we have the most control, i.e. we thoroughly test our programs until they appear to be bug-free. However, it is more realistic to view testing as the aim to reduce as many bugs as possible. So it is possible that a bug lurks deep in the programs implemented, and if one exists it would likely be of the intermittent, and not readily obvious kind. However, the programs did go through extensive testing, including detailed 
comparisons to results of simpler programs executed on subsets of the input data. So we should feel fairly confident in the results obtained.

Hardware problems are not as easy to control. If one is aware that a hardware problem has occurred, then all that need be done is to rerun the particular case. However, it is more likely that hardware errors will go undetected. Still, Lam has stated that it is probable that the plane, if it existed, would have more than one starting point, and hence it is unlikely that a hardware failure would affect all of them given the frequency of such events [3].

However, one can never be absolutely certain that a plane of order 10 doesn't exist solely on the basis of an empirical proof, but with two independent searches reaching the same conclusion, it is less likely than before that a plane was missed. 


\section{Further Research}

Since the non-existence of order 10 was empirically shown with the substantial aid of computers, nothing as significant has been accomplished on the question of existence of finite projective planes. Indeed, we are no closer to determining the validity of the following conjecture [10]:

- The only possible orders for finite projective planes are powers of primes.

There is of course no guarantee that the above "holy grail of finite projective geometry" will ever be settled ${ }^{\Psi}$. But can something be said about other individual composite numbers which aren't excluded as orders of a finite projective plane by the Bruck-Ryser theorem? This next possible order is 12 , and some preliminary work has been undertaken in the last 25 years:

- planes of order 12 with specific collineations have been proven not to exist $[34,35,36,37]$

- a set of 5 mutually orthogonal latin squares of order 12 have been successfully constructed [33];

\footnotetext{
${ }^{\Psi}$ Godel's incompleteness theorem implies that some true statements exist which cannot be proven.
} 
- the error-correcting code $\mathbf{C}$ (binary or ternary) generated by the incidence matrix of a hypothetical projective plane of order 12 has been analyzed [32].

Regarding the last of these items, there are some issues with using the properties of $\boldsymbol{C}$ to determine if a plane of order 12 exists. First off the dimension of $\boldsymbol{C}$ as a binary code cannot be readily determined, hence relations derived from the MacWilliams identities would be incomplete. $\boldsymbol{C}$ as a ternary code has been fully analyzed, but the configurations are too complex to allow a computer attack. Hence at this point, it seems impossible to systematically settle the existence of a plane of order 12.

It may however be possible to use computers to settle another long-standing open problem regarding projective planes. Currently the only know nondesarguesian planes are of prime power order $(e . g 9,16,27$, etc...). It is not known if any non-desarguesian projective plane of prime order exist [10]. It has been determined that for prime orders $2,3,5$, and 7 the only planes that exist are desarguesian (one each up to isomorphism). It is unknown for all prime orders greater or equal to 11 . While it would be preferable to settle the question for all orders $p^{1} \geq 11$, it may be within reach to solve order 11 using a computer ${ }^{\Psi}$.

\footnotetext{
${ }^{\Psi}$ Perhaps if this question is posed as an exercise in class, I may be hooked as was the case with order $10(-)$
} 


\section{Appendix A}

The following table contains detailed numbers for all searches:

\begin{tabular}{|c|c|c|c|c|}
\hline & $M_{15} \mathrm{~s}$ & $M_{16 s^{\prime}}$ & $P_{19 s}$ & Total \\
\hline Nodes & $2,191,912,798$ & $1,302,134,185,498,510$ & $1,213,792,676,025,790$ & $2,515,929,053,437,098$ \\
\hline $\begin{array}{l}\text { Single } \\
\text { core } \\
\text { Time }\end{array}$ & 13989 secs & 182 years & 219 years & 401 years \\
\hline $\begin{array}{l}\text { Multı- } \\
\text { core } \\
\text { Tıme } \\
\text { (15) }\end{array}$ & $N / A^{2}$ & 4441 days & 5322 days & 9763 days \\
\hline$A 1^{3}$ & 1 & 22 & $62^{*}$ & 85 \\
\hline$A 2^{4}$ & 1 & 246,828 & 641,631 & 888,910 \\
\hline$B 1$ & 1 & $1,556,542$ & $64,820,876$ & $66,377,419$ \\
\hline$B 2$ & $79^{4}$ & $318,324,896$ & $113,218,524,289$ & $113,536,849,264$ \\
\hline$B 3$ & $124,126,848$ & $26,622,078,286$ & $9,288,716,655$ & $36,034,921,789$ \\
\hline$B 4$ & 0 & 945,336 & 110,525 & $1,055,861$ \\
\hline Total & $124,126,930$ & $26,943,151,910$ & $122,572,814,038$ & $149,639,796,520$ \\
\hline
\end{tabular}

The search processed approximately $3 \times 10^{8}$ nodes per second ${ }^{5}$.

\footnotetext{
${ }^{1}$ The different $M_{16}$ search technique employed in this thesis resulted in significantly increased processing time when compared to the previous searches done by Carter and Lam

${ }^{2}$ The input to the block extension program is an $A 2$, so this case is not divided on multiple cores

${ }^{3}$ These are unique up to isomorphism

${ }^{*}$ Excludes the $\boldsymbol{P}_{19}$ s which give rise to $\boldsymbol{M}_{16} \mathrm{~s}$

${ }^{4}$ These are unique up to isomorphism

$\Psi$ This number is unique up to isomorphism

${ }^{5}$ The searches were executed on 4 gigabytes RAM, AMD cpu ( $\left.24 \mathrm{gHz}\right)$ mult1-core computers running 64 bit Linux operatıng systems
} 


\section{Appendix B}

The following 56x23 matrix is obtained from the equality ${ }^{\Psi}$ produced when applying the MacWilliams identities on $\boldsymbol{C}$ and its dual code $\boldsymbol{C}^{\perp}$ :

\begin{tabular}{|c|c|c|c|c|c|c|}
\hline$A_{12}$ & $A_{16}$ & $A_{20}$ & $A_{88}$ & $A_{92}$ & $A_{96}$ & \\
\hline 1 & 1 & 1 & 1 & 1 & 1 & $2^{55}-112$ \\
\hline $\mathrm{C}_{99.12}{ }_{110}$ & $\mathrm{C}_{95,16^{110}}$ & $\mathrm{C}_{91,20}{ }^{110}$ & $\mathrm{C}_{23,88^{110}}$ & $C_{13.92^{110}}$ & $C_{15,96}{ }^{110}$ & $-\left(c_{111,0}{ }^{110}+111 c_{11,100^{110}}\right)$ \\
\hline $\mathrm{C}_{99,12^{109}}$ & $\mathrm{C}_{95,16}{ }^{109}$ & $\mathrm{C}_{91,20}{ }^{109}$ & $C_{23,88^{109}}$ & $C_{19,92^{109}}$ & $\mathrm{C}_{15,96^{109}}$ & $-\left(C_{111,0^{109}}+111 C_{11,100}{ }^{109}\right)$ \\
\hline $\mathrm{C}_{99,12^{100}}$ & $\mathrm{C}_{95,16^{100}}$ & $\mathrm{C}_{91,20}{ }^{100}$ & $\mathrm{C}_{23,88}{ }^{100}$ & $C_{19,92}{ }^{100}$ & $c_{15,96}{ }^{100}$ & $2^{55 * 111-\left(c_{111,0} 0^{100}+111 c_{11,100}{ }^{100}\right)}$ \\
\hline$C_{99}, 12^{99}-2^{55}$ & $C_{95,96}{ }^{99}$ & $\mathrm{C}_{91,20^{99}}$ & $C_{23,88^{99}}$ & $\mathrm{C}_{19,92^{99}}$ & $C_{15,96}{ }^{99}$ & $-\left(C_{111,0^{99}}+111 C_{11}, 100^{99}\right)$ \\
\hline $\mathrm{C}_{99}, 12^{96}$ & $C_{95,16}^{96}$ & $\mathrm{C}_{91,20^{96}}$ & $\mathrm{C}_{23,88^{96}}$ & $C_{19,92^{96}}$ & $C_{15,96}{ }^{96} \cdot 2^{55}$ & $-\left(C_{111,0^{96}}+111 C_{11,100^{96}}\right)$ \\
\hline $\mathrm{C}_{99,12^{95}}$ & $\mathrm{C}_{95,16}{ }^{95}-2^{55}$ & $\mathrm{C}_{91,20^{95}}$ & $\mathrm{C}_{23,88^{95}}$ & $C_{19,92}{ }^{95}$ & $c_{15,96}{ }^{95}$ & $-\left(c_{111,0^{95}}+111 C_{11,100^{95}}\right)$ \\
\hline$C_{99,12} 2^{57}$ & $C_{95,16}{ }^{57}$ & $C_{91,20}{ }^{57}$ & $C_{23,88^{57}}$ & $C_{19,92}{ }^{57}$ & $C_{15,96^{57}}$ & $-\left(C_{111,0^{57}}+111 C_{11,100^{57}}\right)$ \\
\hline $\mathrm{C}_{99,12} 56$ & $C_{95,16}{ }^{56}$ & $C_{91,20} 56$ & $\mathrm{C}_{23,88^{56}}$ & $c_{19,92^{56}}$ & $C_{15} .96^{56}$ & $-\left(c_{111,0^{56}}+111 c_{11100^{56}}\right)$ \\
\hline
\end{tabular}

$A_{j}$ represents the coefficients in $C$ 's weight enumerator, i.e. $A_{j}$ is the number of codewords of weight $\mathrm{j}$ in $C . \mathrm{C}_{\mathrm{i}, \mathrm{j}} \mathrm{j}^{\mathrm{k}}$ represents the coefficient of $x^{\mathrm{k}} y^{111-\mathrm{k}}$ in the expansion of $(x+y)^{i}(x-y)^{j}($ where $i+j=111)$. Note that some of these coefficients have more than 30 digits.

$$
\begin{aligned}
& \begin{array}{c}
\Psi_{2}^{55}\left(x^{111}+11 x^{100} y^{11}+\sum_{l=0}^{21} \mathrm{~A}(4 l+12) x^{(99-4 I)} y^{(4 l+12)}+\mathrm{A}(96-4 I)^{(96-4 I)} y^{(4 l+15)}+11 x^{11} y^{100}+y^{111}\right) \\
=
\end{array}
\end{aligned}
$$

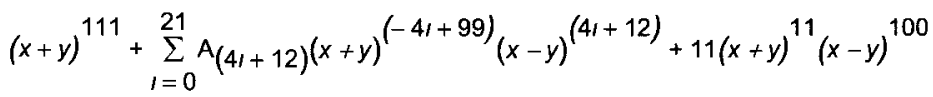




\section{Appendix C}

The following matrix ${ }^{* 1}$ is a row-reduced form of the matrix on the previous page:

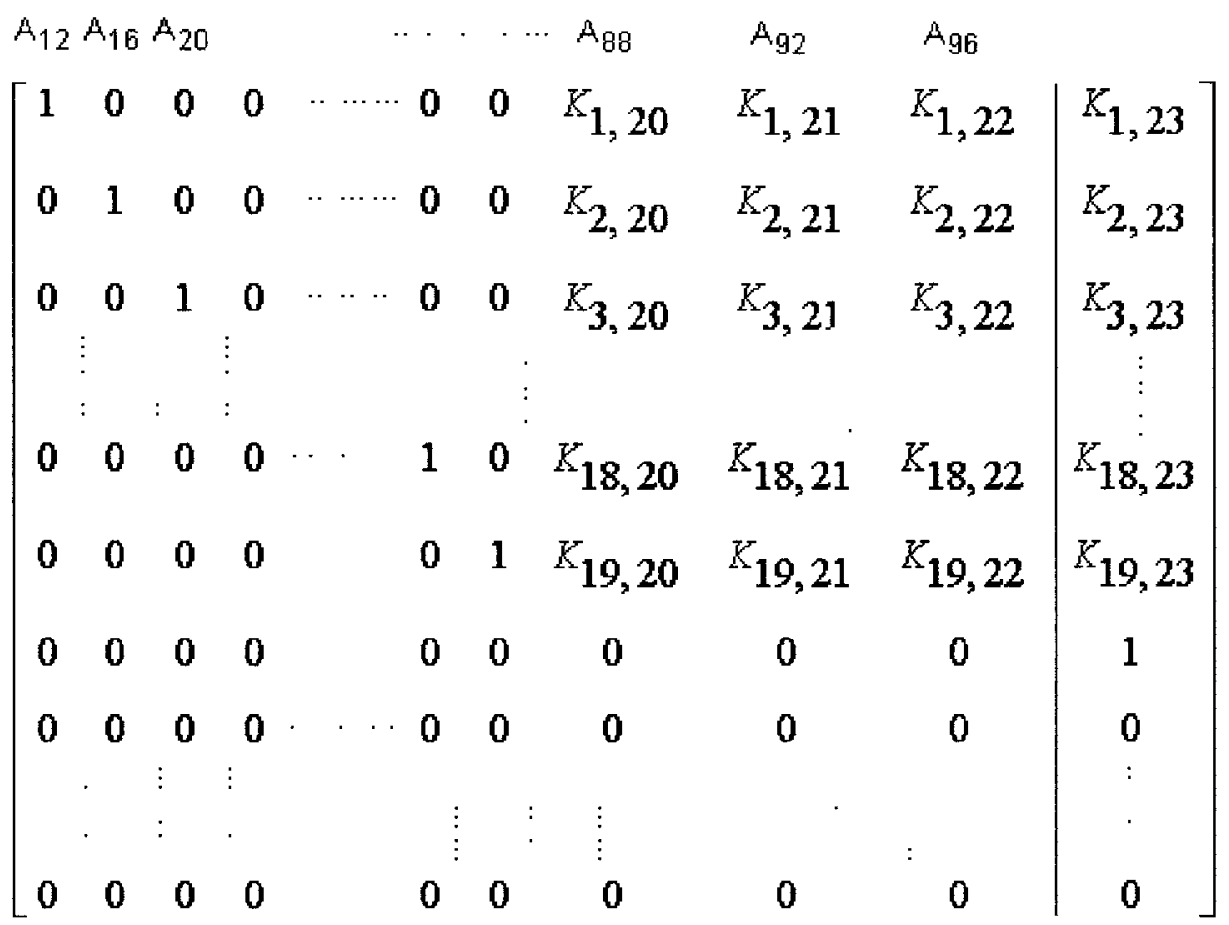

$K_{i, j}$ represents the rational value at the $\mathrm{i}^{\text {th }}$ row and the $\mathrm{j}^{\text {th }}$ column. We simply combine rows 1 and 2 to obtain the desired relation ${ }^{\Psi}$ :

$$
w_{19}=24675+141 w_{12}-27 w_{15}+7 w_{16}
$$

\footnotetext{
"Note that the unusual looking $20^{\text {th }}$ row indicates that the sum of no codewords is the single all-zero vector which must exist in the code $C$.

${ }^{1} \mathrm{~A}_{92}=\mathrm{A}_{19}$ and $\mathrm{A}_{96}=\mathrm{A}_{15}$.

${ }^{\Psi}$ The coefficient for $\boldsymbol{w}_{15}$ seems not to have ever previously appeared in print. This is likely because $\boldsymbol{w}_{15}=0$ was determined before the first printed reference of the above relation expressing $\boldsymbol{w}_{19}$.
} 


\section{Bibliography}

[1] D.R. Stinson, "Combinatorial Designs: Constructions and Analysis", University of Waterloo (2002)

[2] H.S.M Coxeter, "Projective Geometry", Springer-Verlag (1987)

[3] C.W.H. Lam, "The Search for a Finite Projective Plane of Order 10", Concordia University (1996)

[4] E.F. Assmus, Jr. and H.F. Mattson, Jr., "On the Possibility of a Projective Plane of Order 10", Cambridge Research Laboratories (1970)

[5] J. MacWilliams, N.J.A. Sloane, J.G Thompson, "On the existence of a projective plane of order 10", J. Comb Theory (1973)

[6] J.L. Carter, "On the Existence of a Projective Plane of Order Ten", Ph.D. Thesis University of California (1974)

[7] C.W.H. Lam, L. Thiel, S. Swiercz, "The Non-Existence of Finite Projective Planes of Order 10", Concordia University (1989) 
[8] M. Hall, Jr., "Configurations in a plane of order 10", Ann. Discrete Math (1980)

[9] C.L. Mallows and N.J.A Sloane, "Weight Enumerators of self-orthogonal Codes", Discrete Math. (1974)

[10] C.J. Colbourn, J.H. Dinitz, "Handbook of Combinatorial Designs", Chapman and Hall/CRC (2006)

[11] R. H. Bruck and H. J. Ryser, "The non-existence of certain finite projective planes", Can. J. Math., Vol. 1(1949)

[12] L. Euler, "Recherches sur une nouvelle espèce de quarrés magiques", Verh.

Zeeuwsch. Genootsch. Wetensch. Vlissengen Vol 9 (1782)

[13] R.C. Bose, "On the application of Galois fields to the problem of construction of hyper-Graeco-Latin squares", Sankhyã, Vol 3 (1938)

[14] E. T. Parker, " Construction of some sets of mutually orthogonal Latin squares," Proc. Amer. Math. Soc., Vol. 10(1959)

[15] S.H. Whitesides, "Projective planes of order 10 have no collineations of order 11", Utilitas Math. (1976) 
[16] S.H. Whitesides, "Collineations of projective planes of order 10", J. Comb.

Theory, Ser. A (1979)

[17] R.P. Anstee, M. Hall, Jr. and J.G. Thompson, "Planes of order 10 do not have a collineation of order 5", J. Comb. Theory, Ser. A, 29 (1980)

[18] Z. Janko, T. van Trung, "Projective planes of order 10 do not have a collineation of order 3", J. Reine Angew. Math., Vol. 325 (1981)

[19] K.H. Rosen, "Discrete Mathematics and its applications", McGraw-Hill (1999)

[20] D.E. Knuth, "Estimating the Efficiency of Backtrack Programs", Mathematics of Computations, Vol. 29 (1975)

[21] F.J MacWilliams, N.J.A. Sloane, “The theory of Error-Correcting Codes", North-Holland Publishing Company (1977)

[22] D.W. Erbach, "Code theoretic evidence concerning the plane of order 10", Dissertation, Cambridge University (1976)

[23] J.G. Thompson, "Ovals in a projective plane of order 10", London Math.

Society, Lecture Note Series 52 (1981) 
[24] B.D. Mackay, "Nauty User's Guide v2.4", Australian National University (2007)

[25] D.J. Roy, "Proving $w_{15}=0$ in a Hypothetical Projective Plane of order 10 ", Course Project for CSI 5165, Ottawa University (2005)

[26] C.W.H. Lam, L. Thiel, S. Swiercz, "The Non-Existence of Code Words of Weight 16 in a Projective Plane of Order 10", J. Combinatorial Theory Ser. A (1986)

[27] C.W.H. Lam, S. Crossfield, L. Thiel, "Estimates of a computer search for a projective plane of order 10", Congressus Numerantium Vol. 48 (1985)

[28] C.W.H. Lam, L. Thiel, S. Swiercz, "The Nonexistence of ovals in a projective plane of order 10", Discrete Mathematics, Vol. 45 (1983)

[29] C.W.H. Lam, L. Thiel, S. Swiercz, "Estimates of a computer search for a projective plane of order 10", Congressus Numerantium 48 (1985) 
[30] L. Thiel, C. Lam, S. Swiercz, "Using a CRAY-1 to perform a backtrack search", Proc. Of the Second International Conference on Supercomputing, Vol. III (1987)

[31] C.W.L. Garner, "Course Notes for Foundations of Geometry", Carleton University (2002)

[32] M. Hall, Jr. and J. Wilkinson, "Ternary and Binary Codes for a Plane of Order 12", J. of Combinatorial Theory, Ser. A (1984)

[33] D.M. Johnson, A.L. Dulmage and N.S.Mendelshon, "Orthomorphism of groups and orthogonal Latin squares", Canadian Journal of Mathematics (1961)

[34] Z. Janko, T. van Trung, "The full collineation group of any projective plane of order 12 is a $\{2,3\}$-group", Geometriae Dedicata, (1982)

[35] Z. Janko, T. van Trung, "Projective planes of order 12 do not have a nonabelian group of order 6 as a collineation group", J. Reine Angew. Math (1981)

[36] Z. Janko, T. van Trung, "Projective Planes of Order 12 Do Not Have a Four Group as a Collineation Group", J. Combinatorial Theory, Ser. A (1982) 
[37] K. Horvatic-Baldasar, E. Kramer, I. Matulic-Bedenic, "Projective planes of order 12 do not have an abelian group of order 6 as a collineation group", Punime Mat (1986)

[38] S. Dow, "An improved bound for extending partial projective planes", Discrete Mathematics, Vol. 45 (1983)

[39] S. Dow, "On the number of lines in a maximal partial projective plane", Congressus Numerantium 39 (1983)

[40] S. H. Suyu, P. J. Marshall, M. W. Auger, S. Hilbert, R. D. Blandford, L. V. E. Koopmans, C. D. Fassnacht and T. Treu, "Dissecting the Gravitational Lens B1608+656. II. Precision Measurements of the Hubble Constant, Spatial Curvature, and the Dark Energy Equation of State", The Astrophysical Journal, 2010; 711 (1): 201 DOI: 10.1088/0004-637X/711/1/201 